\title{
Benign T cells drive clinical skin inflammation in cutaneous T cell lymphoma
}

Pablo Vieyra-Garcia, ${ }^{1,2}$ Jack D. Crouch, ${ }^{1}$ John T. O'Malley, ${ }^{1}$ Edward W. Seger, ${ }^{1}$ Chao H. Yang, ${ }^{1}$ Jessica E. Teague, ${ }^{1}$ Anna Maria Vromans, ${ }^{1}$ Ahmed Gehad, ${ }^{1}$ Thet Su Win, ${ }^{1}$ Zizi Yu, ${ }^{3}$ Elizabeth L. Lowry, ${ }^{1}$ Jung-Im Na, ${ }^{1,4}$ Alain H. Rook, ${ }^{5}$ Peter Wolf, ${ }^{2}$ and Rachael A. Clark'

'Department of Dermatology, Brigham and Women's Hospital, Harvard Medical School, Boston, Massachusetts, USA. ${ }^{2}$ Research Unit for Photodermatology, Department of Dermatology and Venereology, Medical University of Graz, Graz, Austria. ${ }^{3}$ Harvard Medical School, Boston, Massachusetts, USA. ${ }^{4}$ Department of Dermatology, Seoul National University Bundang Hospital, Seongnam, South Korea. ${ }^{5}$ Department of Dermatology, Perelman School of Medicine, University of Pennsylvania, Philadelphia, Pennsylvania, USA.

Psoralen plus UVA (PUVA) is an effective therapy for mycosis fungoides (MF), the skin-limited variant of cutaneous T cell lymphoma (CTCL). In low-burden patients, PUVA reduced or eradicated malignant $T$ cells and induced clonal expansion of $C D 8^{+} T$ cells associated with malignant $T$ cell depletion. High-burden patients appeared to clinically improve but large numbers of malignant $T$ cells persisted in skin. Clinical improvement was linked to turnover of benign T cell clones but not to malignant T cell reduction. Benign T cells were associated with the Th2-recruiting chemokine CCL18 before therapy and with the Th1-recruiting chemokines CXCL9, CXCL10, and CXCL11 after therapy, suggesting a switch from Th2 to Th1. Inflammation was correlated with OX4OL and CD4OL gene expression; immunostaining localized these receptors to CCL18-expressing c-Kit ${ }^{+}$dendritic cells that clustered together with $\mathrm{CD} 40^{+} \mathrm{OX} 40^{+}$benign and $\mathrm{CD} 40^{+} \mathrm{CD} 40 \mathrm{~L}^{+}$malignant $\mathrm{T}$ cells, creating a proinflammatory synapse in skin. Our data suggest that visible inflammation in CTCL results from the recruitment and activation of benign $T$ cells by c-Kit' $\mathrm{OX} 40 \mathrm{~L}^{+} \mathrm{CD} 40 \mathrm{~L}^{+}$dendritic cells and that this activation may provide tumorigenic signals. Targeting c-Kit, OX40, and CD40 signaling may be novel therapeutic avenues for the treatment of MF.

Conflict of interest: The authors have declared that no conflict of interest exists.

License: License: This work is licensed under the Creative Commons Attribution 4.0 International License. To view a copy of this license, visit http://creativecommons.org/ licenses/by/4.0/.

Submitted: August 14, 2018 Accepted: December 5, 2018 Published: January 10, 2019

\section{Reference information:} JCI Insight. 2019;4(1):e124233. https://doi.org/10.1172/jici. insight.124233.

\section{Introduction}

Cutaneous T cell lymphomas (CTCLs) are a heterogeneous collection of non-Hodgkin's lymphomas derived from $\mathrm{T}$ cells tropic for the skin. Unlike most nodal non-Hodgkin's lymphomas, approximately $75 \%$ of primary cutaneous lymphomas are T cell derived. CTCL patients have inflammatory skin lesions infiltrated by both malignant and benign infiltrating $\mathrm{T}$ cells, and variable involvement of the blood, lymph nodes, and visceral organs. In mycosis fungoides (MF), the most common subtype of CTCL, malignant T cells are confined to inflammatory skin lesions (1). Phenotypic studies have suggested that $\mathrm{MF}$ is a cancer of nonrecirculating, skin-resident memory $\mathrm{T}$ cells $(2,3)$. Twenty percent of MF patients develop progressive, often lethal skin disease (4). Even in patients with indolent MF, lymphomatous involvement of the skin is lifelong and disease is incurable in the absence of stem cell transplantation. A better understanding of the pathogenesis of MF is needed to develop therapies that can cure or halt progression of the disease.

Psoralen plus ultraviolet A (UVA) light (PUVA) is an immunomodulatory therapy for skin disease in which patients ingest or topically apply 8-methoxypsoralen and are exposed to UVA light. PUVA is an effective treatment for MF and for inflammatory and autoimmune disorders including psoriasis, vitiligo, and graft-versus-host disease (5-7). PUVA induces remission in $72 \%$ of MF patients; remission can last up to 10 years with maintenance therapy and PUVA is effective in MF patients who fail to respond to narrowband UVB $(1,8,9)$. The mechanisms of action of PUVA are poorly understood and it is not clear why it is effective in treating both a malignant lymphoma and inflammatory and autoimmune disorders such as psoriasis and vitiligo. To address these unanswered questions, we carried 
out translational studies using high-throughput sequencing (HTS) of T cell receptors (TCRs), NanoString-based gene expression profiling, and multiplex immunostaining of biopsies of MF skin lesions before and after PUVA therapy.

\section{Results}

PUVA is an effective therapy for MF that can eradicate malignant $T$ cells in patients with low initial tumor burden. Fifteen patients with stage IA-IIA mycosis fungoides were treated with PUVA twice weekly for 12 to 15 weeks. Clinical severity of disease was assessed in a single index lesion using the Composite Assessment of Index Lesion Severity (CAILS) scoring system and across the entire body surface area using the Modified Severity-Weighted Assessment Tool (mSWAT) scoring system (Figure 1, A and B). Skin biopsies of a single index lesion were obtained before and after therapy and translational studies were carried out using HTS of the TCR CDR3 regions, NanoString-based gene expression profiling, and multiplex immunostaining (Figure 1C). Fourteen out of 15 patients showed reduced skin inflammation in the index lesion and reductions in overall burden of disease after PUVA therapy (Figure 1, D-F). T cell numbers as measured by HTS were significantly reduced after therapy (Figure $1 G$ ). However, the changes in the malignant $\mathrm{T}$ cell frequency after therapy varied among patients (Figure $1 \mathrm{H}$ ). There was a wide range of malignant $\mathrm{T}$ cell frequencies before therapy but patients clustered into groups that had high or low malignant $\mathrm{T}$ cell frequencies after therapy (Figure 1I). We separated patients into different groups based on their initial malignant $\mathrm{T}$ cell frequency and found that responses were significantly different in these groups (Figure 1, J-L). Fifty percent of patients with less than $10 \%$ malignant $\mathrm{T}$ cell clones experienced complete eradication of the malignant $\mathrm{T}$ clone after PUVA. Patients with 10\%-20\% malignant T cells in skin had a mean $97 \%$ reduction in the frequency of malignant cells. However, patients with greater than $20 \%$ malignant $\mathrm{T}$ cells experienced a mean $1.5 \%$ increase in the frequency of malignant $\mathrm{T}$ cells. To study this in more detail, we measured the absolute number of malignant and benign $\mathrm{T}$ cell numbers in skin in low-burden $(\leq 20 \%)$ and high-burden $(>20 \%)$ patients by HTS. The absolute numbers (genomes per $100 \mathrm{ng}$ of DNA) of total T cells, malignant T cells, and benign $\mathrm{T}$ cells were all significantly reduced in low-burden patients but varied widely and were not significantly different in high-burden patients (Figure 1, M and N). In summary, PUVA therapy effectively reduced or eliminated malignant $\mathrm{T}$ cells in low-burden but not high-burden patients.

Visible inflammation does not reflect malignant $T$ cell burden and reduced inflammation is linked to turnover of benign $T$ cells. We were surprised to find that clinical responses were not significantly different in high-burden and low-burden patients (Figure 2A). High-burden patients had reduced clinical inflammation scores despite the high frequencies of malignant $\mathrm{T}$ cells in skin after therapy (Figure 2, B-D). Patient 4 experienced complete clearance of all skin disease clinically but malignant $\mathrm{T}$ cells in this patient increased from $51 \%$ to $69 \%$ after treatment (Figure 2, B and C). Similar responses were seen in 3 other high-burden patients (Figure 2D). Evaluation of the benign T cells by HTS showed that there were marked shifts in the benign $\mathrm{T}$ cell populations in high-burden patients after therapy (Figure 2, C and D). Largely nonoverlapping populations of benign $\mathrm{T}$ cells, identified by their unique antigen receptors, were present before and after therapy. We correlated improvements in clinical exam scores with various $\mathrm{T}$ cell parameters to identify aspects that correlated with reduced visible inflammation. Improvement in inflammation correlated with turnover of the benign $\mathrm{T}$ cell populations (Figure $2 \mathrm{H}$ [mSWAT] and Supplemental Figure 1D [CAILS]; supplemental material available online with this article; https://doi.org/10.1172/jci. insight.124233DS1) but not with reductions in the number of total $\mathrm{T}$ cells, malignant $\mathrm{T}$ cells, or total benign T cells (Figure 2, E-G [mSWAT], and Supplemental Figure 1, A-C [CAILS]). These data suggest that visible inflammation was not driven by high numbers of benign or malignant $\mathrm{T}$ cells in skin but instead by a specific population of benign $\mathrm{T}$ cell clones present before therapy, identifiable by their unique antigen receptors, that was eliminated by PUVA. PUVA also recruited a new population of benign $\mathrm{T}$ cells bearing distinct antigen receptors into skin.

Benign T cells have distinct gene association profiles before and after therapy and PUVA induces a shift from Th2 to Th1 chemokine association. We studied the expression of a set of inflammation-related genes in skin biopsies before and after PUVA therapy by NanoString. Loss of CD7 on malignant T cells can be a useful marker for distinguishing benign from malignant $\mathrm{T}$ cells in $\mathrm{MF}(10,11)$. To identify genes in the codeset associated with benign $\mathrm{T}$ cells, we correlated gene expression levels of CD7, which should be expressed on benign $\mathrm{CD}^{+}$and $\mathrm{CD}^{+} \mathrm{T}$ cells but lost on malignant $\mathrm{T}$ cells, with genes across the data set. CD7 gene expression correlated with the number of benign T cells by HTS, validating this approach (Supplemental Figure 2). To 
A PUVA: psoralen plus UVA

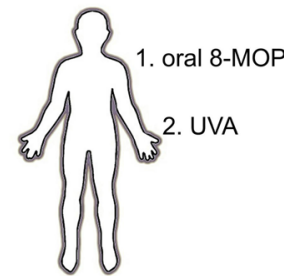

B

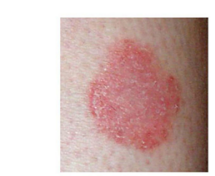

Composite Assessment of Index Lesion Severity
Clinical assessment mSWAT

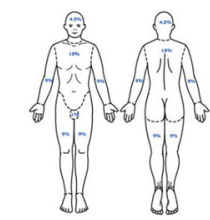

Modified Severity-Weighted Assessment Tool
C

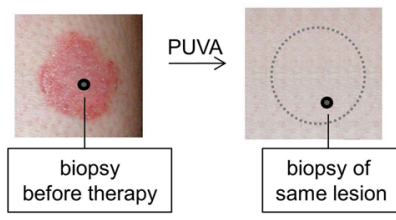

- TCR sequencing

- NanoString gene expression profiling

- Immunostaining \& multispectral imaging
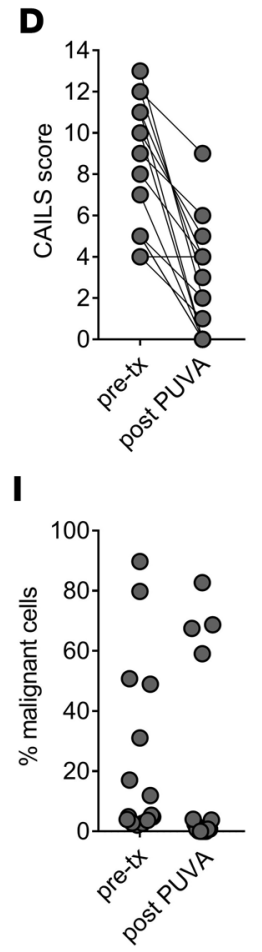

E

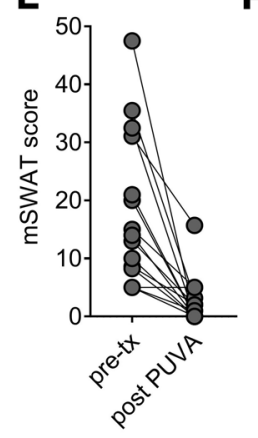

$\mathbf{F}$

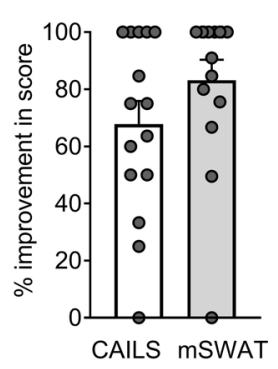

G
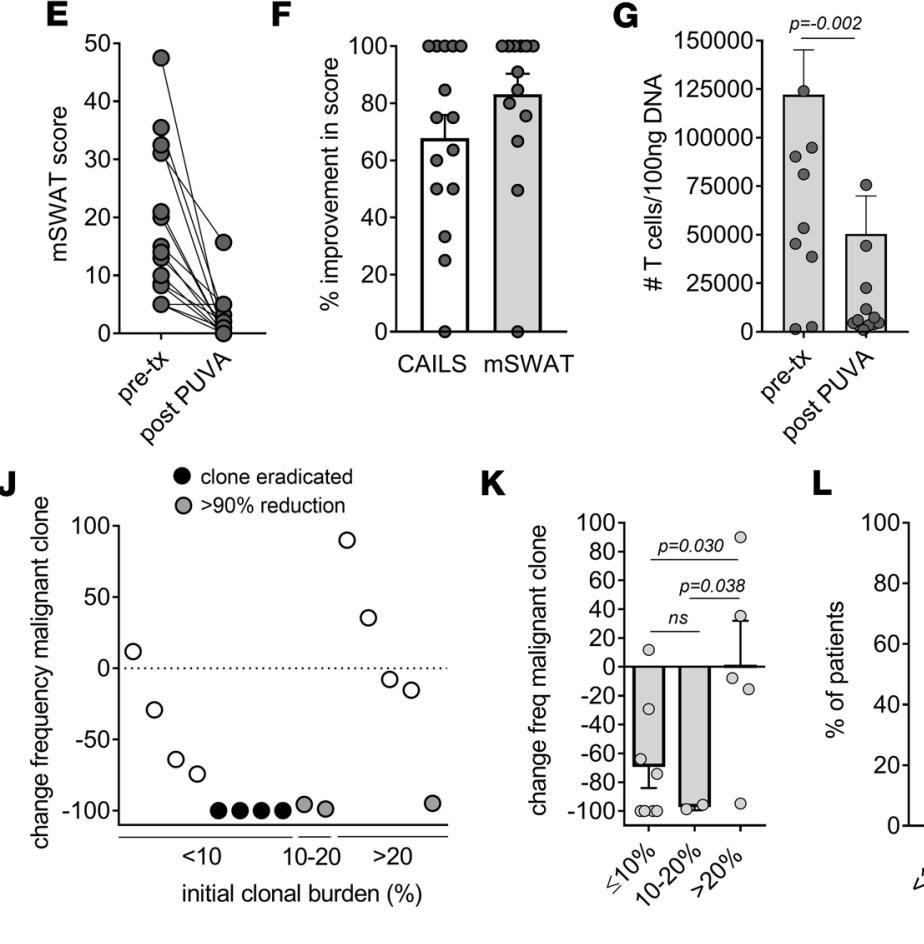

$\mathbf{K}$

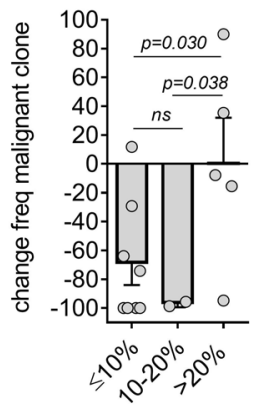

$\mathbf{L}$
H

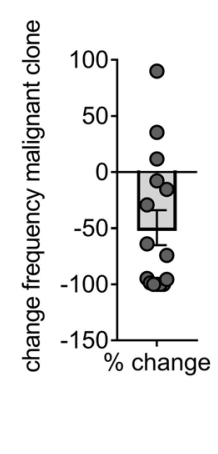

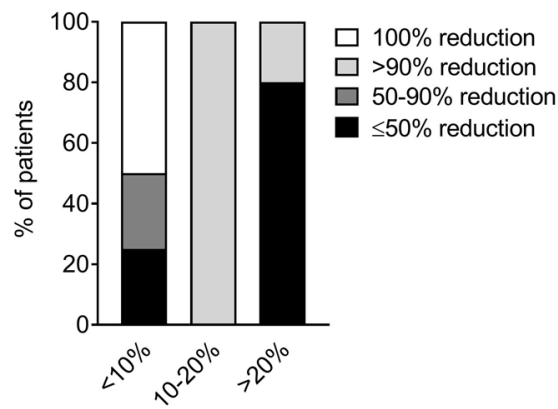

N high burden (>20\%)

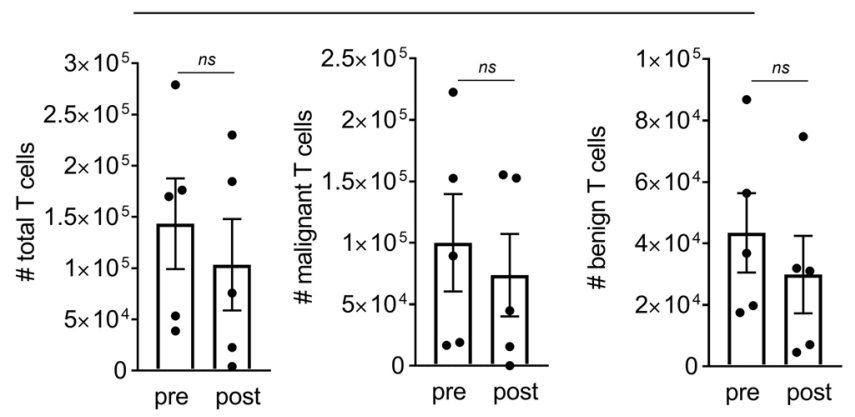

Figure 1. PUVA is an effective therapy for mycosis fungoides that can eradicate malignant $T$ cells in patients with low initial tumor burden. (A-C) Design of the clinical and translational studies. (A) Fifteen patients were treated with oral 8-methoxypsoralen (8-MOP) and exposed to UVA light. (B) Severity of disease was assessed in a single index lesion using the CAILS scoring system and across the entire body surface area using the mSWAT scoring system. (C) Skin biopsies of a single index lesion were obtained before and after therapy and were studied by HTS, NanoString-based gene expression profiling, and multiplex immunostaining. (D-F) All patients improved clinically with respect to index lesion severity (D) and overall burden of disease (E). Pre-tx, pretreatment. Individual patients ( $\mathbf{D}$ and $\mathbf{E})$ and aggregate clinical scores (F) are shown. (G) The number of total T cells in skin as assessed by HTS was significantly reduced after therapy but $\mathbf{( H )}$ changes in the malignant T cell frequency varied widely among patients. (I-L) Patients with a low initial malignant $T$ cell burden had the best clearance of malignant T cells. (I) There was a wide range of initial malignant $\mathrm{T}$ cell frequencies before therapy but patients tended to cluster into either high or low residual malignant T cells groups after therapy. (J-L) Patients with a low initial malignant T cell frequency ( $<10 \%$ or $10 \%-20 \%)$ had the greatest depletion of malignant T cells. The change in the malignant T cell frequency for each patient (J), the mean reduction in malignant $T$ cell frequency in each group (K), and the percentage of patients in each group with the indicated levels of malignant T cell reductions (L) are shown. (M and $\mathbf{N}$ ) 
PUVA reduced the absolute number of benign and malignant T cells in skin from low-burden but not high-burden patients. The absolute number of total, malignant, and benign T cells (T cell genomes/100 ng of DNA) in skin for low-burden (M) and high-burden ( $\mathbf{N}$ ) patients are shown. The mean and SEM (error bars) are shown. Differences between 2 sample groups were detected using the 1-tailed Wilcoxon-Mann-Whitney test $(\alpha=0.05)$. For comparisons of multiple groups, a Kruskal-Wallis 1-way analysis of variance with a Bonferroni-Dunn post hoc test for multiple means testing was used ( $\alpha=0.05)$. All reported $P$ values are adjusted for multiple comparison testing.

identify genes selectively associated with benign $\mathrm{CD} 8^{+} \mathrm{T}$ cells, we correlated expression of the CD8A gene with other genes in the data set. A list of CD7- and CD8-associated genes and their $r$ and $P$ values is included in Supplemental Tables 1 and 2. Genes associated with total benign $\mathrm{T}$ cells had only 1 gene in common before and after PUVA, suggesting a shift in benign $\mathrm{T}$ cell gene expression profiles. CD8-associated genes were completely distinct before and after therapy. The number of benign $\mathrm{T}$ cells before therapy was tightly correlated with expression of CCL18, a Th2-recruiting cytokine $\left(r^{2}=0.856, P=0.001\right)$, but this association was lost after therapy (Figure 3B). After therapy, the number of benign $\mathrm{T}$ cells instead correlated closely with levels of the Th1-recruiting chemokines CXCL9, CXCL10, and CXCL11 $\left(r^{2}=0.989,0.905,0.987 ; P\right.$ $<0.0001, P=0.0035, P<0.0001$, respectively; Figure 3B). These findings suggest a shift in benign $\mathrm{T}$ cell gene expression before and after therapy. Taken together with HTS results, these findings show that distinct populations of $\mathrm{T}$ cells with differing antigen receptors and gene expression profiles are present before and after PUVA therapy and that PUVA may induce a shift in benign T cells from Th2 to Th1.

c-Kit dendritic cells produce CCL18 in MF. Expression of CCL18, a chemokine that specifically recruits Th2 cells, is known to be increased in CTCL skin lesions and correlates with disease progression (12, 13). In our data set, CCL18 was expressed at levels 115 -fold higher than in healthy skin $(P=0.009)$, was reduced after therapy, and was expressed at highest levels in low-burden patients, in whom benign T cells outnumbered malignant $\mathrm{T}$ cells (Supplemental Figure 3). A previous study suggested that CCL18 may be produced by M2 macrophages in CTCL skin lesions (12). However, CCL18 levels in our data set did not correlate with CD68, a pan-macrophage marker, or with CD163, a marker of M2-like macrophages (Supplemental Figure 3). We immunostained MF skin lesions to identify the cell type expressing CCL18 (Figure 4). CCL18 was expressed at high levels but did not colocalize with CD163. Instead, CCL18 immunostaining was specifically localized to c-Kit-expressing cells in the dermis (Figure 4, B and C). These cells lacked tryptase and expressed CD11c (Figure 4D and Supplemental Figure 4), confirming their identify as c-Kit ${ }^{+}$dendritic cells, a cell type induced by Th2/Th17-inducing stimuli known to impair Th1 responses (14). Our studies suggest that $\mathrm{c}-\mathrm{Kit}^{+}$dendritic cells may play a critical role in MF by producing CCL18, a chemokine that recruits benign, infiltrating Th2 cells.

Skin inflammation does not reflect malignant $T$ cell number or frequency but inflammation is related to $O X 40 L$ and CD40L, 2 genes associated with malignant T cells. Our HTS studies showed that improvement in clinical inflammation after PUVA was linked to benign $\mathrm{T}$ cell turnover but not to malignant $\mathrm{T}$ cell depletion. To further examine how malignant $\mathrm{T}$ cell numbers relate to skin inflammation, we correlated CAILS scores of index lesions with the absolute number and relative percentages of malignant $\mathrm{T}$ cells as measured by HTS in the pretreatment skin lesions of MF patients in 3 separate clinical cohorts from 3 different institutions (Figure 5, A and B). Fifteen patients from our University of Graz PUVA cohort, 16 patients from an irradiation versus topical steroids cohort from the Dana-Farber/Brigham and Women's Cancer Center, and 10 patients from a phase I trial of topical resiquimod at the University of Pennsylvania (15) were studied. There were no correlations between the absolute number or relative percentage of malignant $\mathrm{T}$ cells in skin and visible skin inflammation, as measured by CAILS scores (Figure 5, A and B).

Despite no direct link, malignant $\mathrm{T}$ cells must somehow drive the skin inflammation associated with CTCL. We identified genes that were associated with visible skin inflammation by correlating pretreatment CAILS scores with other genes across the data set in the PUVA cohort. Nine genes were strongly associated with CAILS scores of clinical inflammation (Figure 5C). Three of these genes, CD3G, CCR4, and CD5, are widely expressed by both benign and malignant $\mathrm{T}$ cells in skin. Two genes, TNFSF4 (OX40L) and CD40 ligand (CD40L), were notable because they correlated with malignant $\mathrm{T}$ cell numbers (Figure $5 \mathrm{D}$ and Supplemental Table 3) but not with benign T cell subsets (Supplemental Tables 1 and 2). TNFRSF4 (OX40), the receptor for OX40L, was strongly associated with benign T cells before but not after PUVA (Figure 5D and Supplemental Table 1), suggesting a possible interaction between benign and malignant T cells. CD40L was strongly associated with malignant T cell number $(r=0.932, P=0.002)$ and its receptor $\mathrm{CD} 40$ was associated with $\mathrm{CD} 4^{+} \mathrm{T}$ cell and macrophage (CD68 and CD163) but not dendritic cell (CD11c, CCL13, CCL17, 

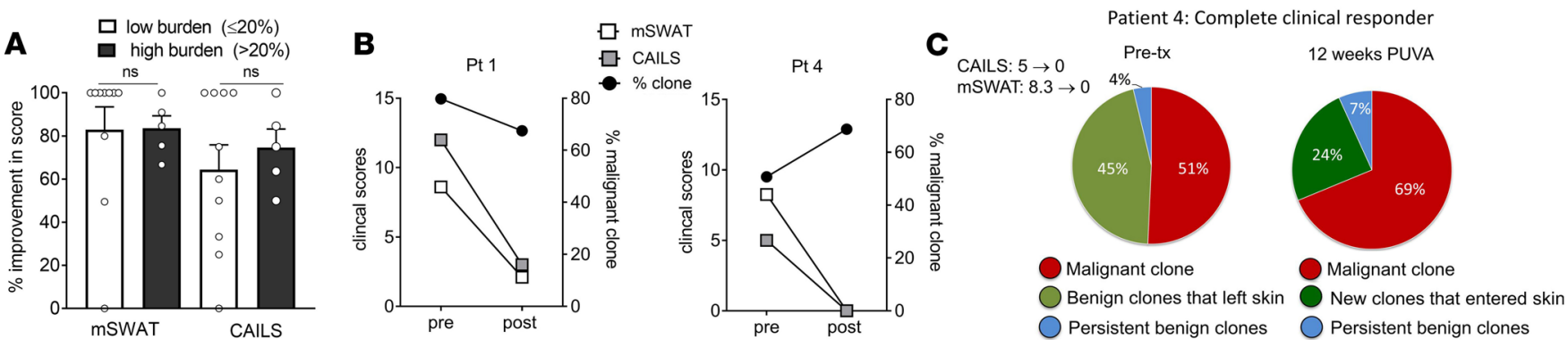

Pt 1: 75\% improved CAILS, 76\% improved mSWAT

Pt 2: 50\% improved CAILS, 91\% improved mSWAT

Patient 3: 64\% improved CAILS, 67\% improved mSWAT

D

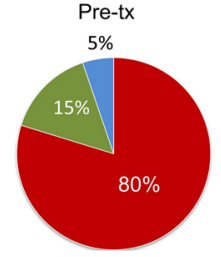

12 weeks PUVA

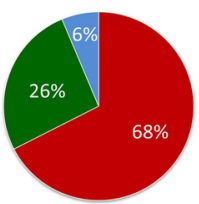

Pre-tx

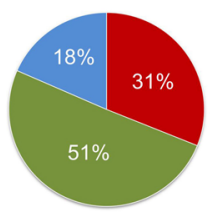

E

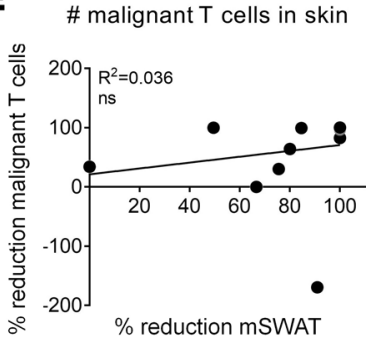

F

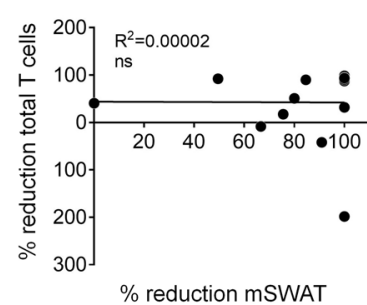

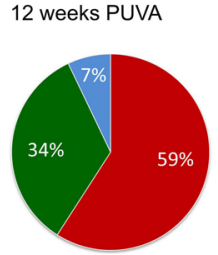

G

G $\quad$ \# benign T cells in skin

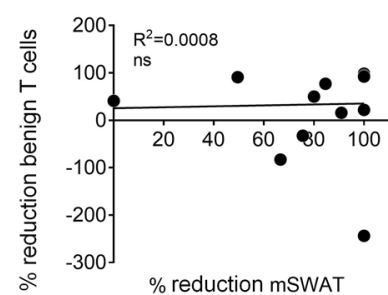

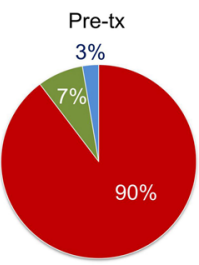

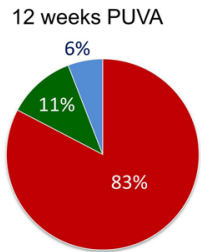

H

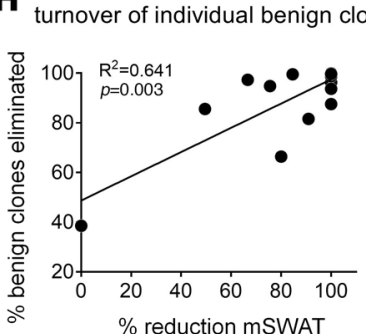

Figure 2. Visible inflammation does not reflect malignant T cell burden and reduced inflammation is linked to turnover of benign T cells. (A) Clinical exam scores in high- and low-burden patients were not significantly different. (B) Two patients are shown in whom visible inflammation (clinical exam scores) improved but the malignant T cell clone remained high after treatment (patient 1,68\%; patient 4, 69\%) or even increased (patient 4). (C) Malignant T cell frequency remained high after treatment despite the presence of large numbers of malignant T cells in skin in patient 4, a complete clinical responder. The unique TCR CDR3 sequences of each nonmalignant T cell clone were used to identify which benign T cells persisted after therapy (blue), were eliminated from skin (light green), or were recruited to skin (dark green) after therapy. Persistent benign clones were benign T cell clones that were present in skin both before and after PUVA therapy. (D) Additional patients are shown in whom the malignant T cell burden remained high after therapy despite improvement in clinical inflammation exam scores. (E-H) Improvement in inflammation is correlated with a shift in the benign T cell population but not with depletion of malignant T cells. Improvement in inflammation (mSWAT) did not correlate with reductions in the number of malignant T cells (E), total T cells (F), or benign T cells (G). However, the loss of specific T cell clones from skin and recruitment of a second, distinct T cell population was correlated with reduced inflammation as assessed by mSWAT $(\mathbf{H})$ and CAILS scores (Supplemental Figure 1). Differences between 2 sample groups were detected using the 1-tailed Wilcoxon-Mann-Whitney test $(\alpha=0.05)$. For correlations, a Pearson's correlation coefficient with a 2-tailed $P$ value is reported.

CCL22, and HSD11B1) markers (Figure 5D and Supplemental Figure 7). These results suggest that interactions between malignant $\mathrm{T}$ cells, benign $\mathrm{T}$ cells, and a nonlymphocytic cell type may drive visible inflammation. The antigen-presenting cell-specific (APC-specific) costimulatory gene CD80 was strongly associated with benign $\mathrm{T}$ cells, supporting the hypothesis that benign $\mathrm{T}$ cells may cluster with a nonlymphoid cell type.

OX40 is expressed by benign T cells and OX40L is expressed by $c$-Kit dendritic cells in MF. Stimulation of OX40 on the surface of $\mathrm{T}$ cells by OX40L drives chronic $\mathrm{T}$ cell activation and cytokine production (16). We immunostained MF skin lesions for OX40 and OX40L to explore the hypothesis that visible inflammation may be driven in part by OX40/OX40L-induced activation of benign T cells. Malignant and benign T cells can be discriminated by costaining for $\mathrm{CD} 3$ and the variable $(\mathrm{V})$ region of the TCR $\beta$ chain (TCR V $\beta$ ) expressed by the malignant $\mathrm{T}$ cell clone, as identified by HTS (Figure 6A) (17). OX40 was specifically and selectively expressed by benign $\mathrm{T}$ cells (Figure $6, \mathrm{~B}$ and $\mathrm{C}$ ), in agreement with the gene association studies. However, OX40L was expressed specifically and at high levels by c-Kit-expressing dermal cells but not by $\mathrm{T}$ cells nor by CD163-expressing macrophages (Figure 6, D and E, and Supplemental Figure 10). OX40L ${ }^{+}$ cells lacked tryptase and expressed CD11c, confirming their identify as c-Kit ${ }^{+}$dendritic cells (Figure $6 \mathrm{~F}$ and 
A

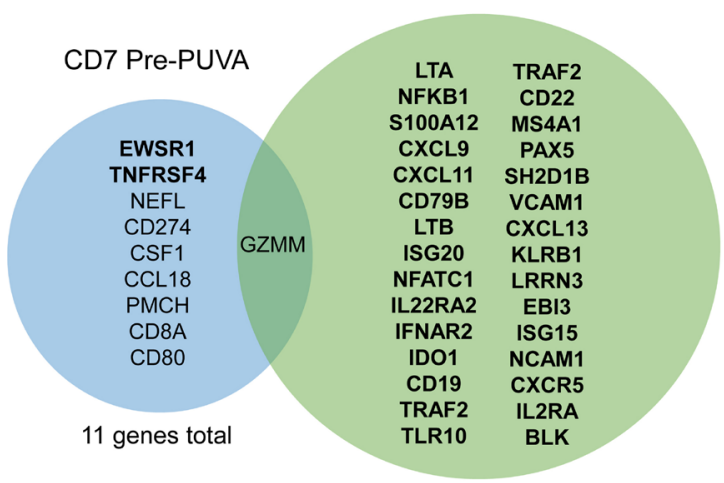

85 genes total

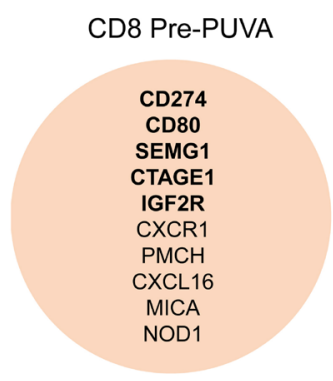

24 genes total
CD8 Post-PUVA

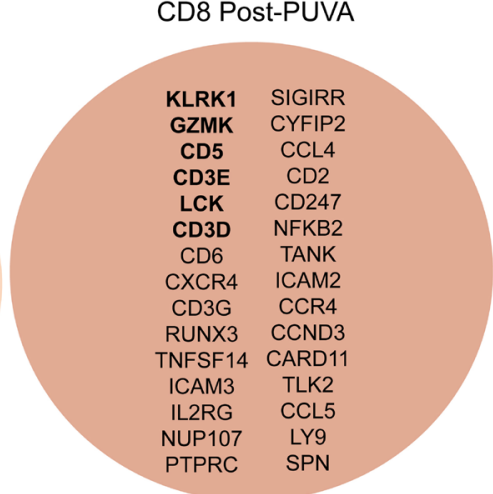

46 genes total
B

$\mathrm{T}_{\mathrm{H}} 2$

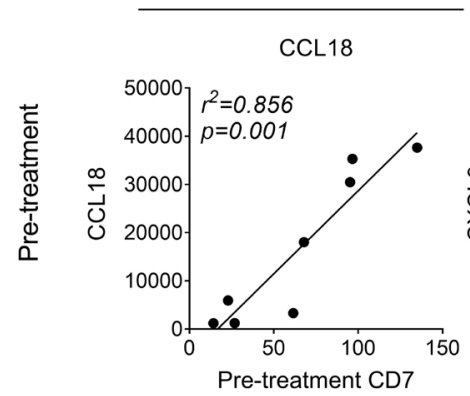

CCL18

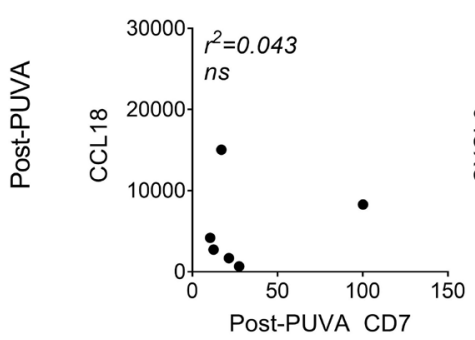

CCL18

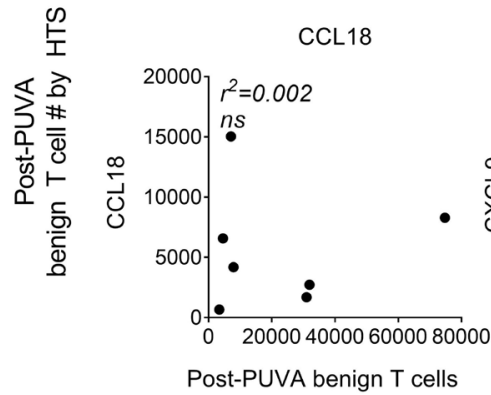

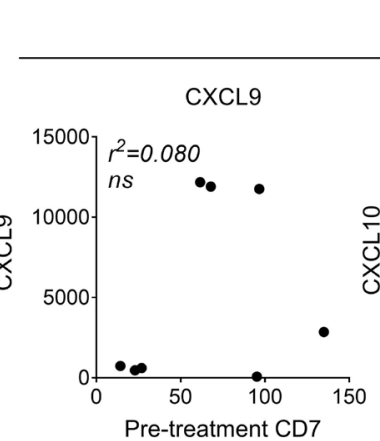

CXCL9

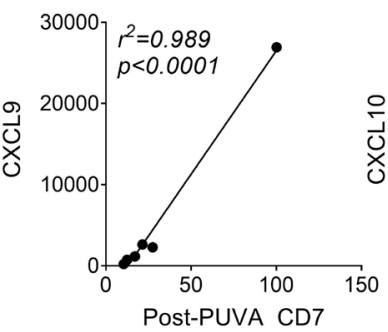

CXCL9

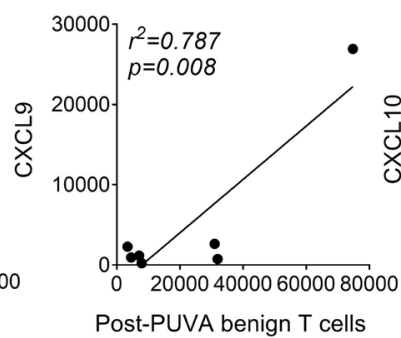

$\mathrm{T}_{\mathrm{H}} 1$

CXCL10

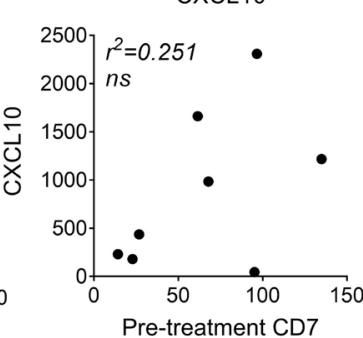

CXCL10

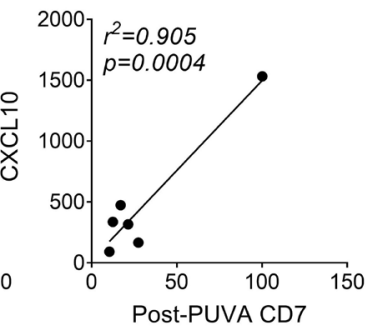

CXCL10

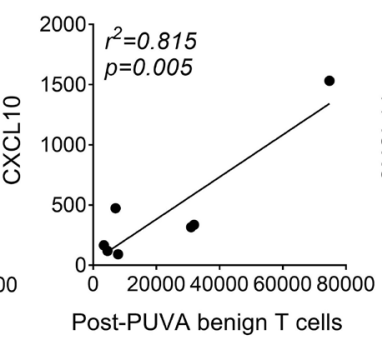

CXCL11

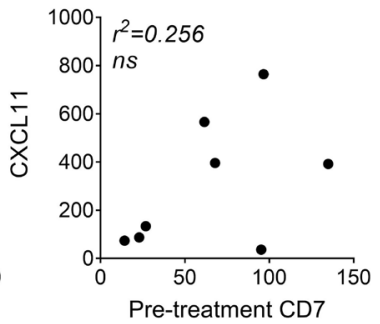

CXCL11

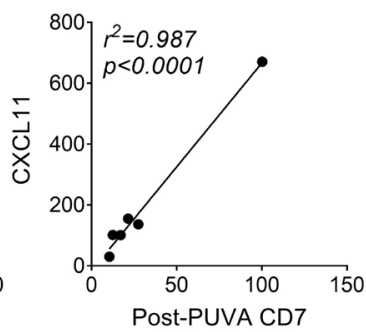

CXCL11

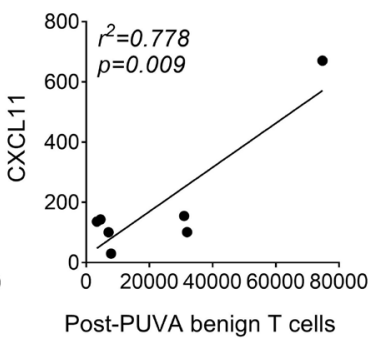

Figure 3. Benign T cells have distinct gene association profiles before and after therapy and PUVA induces a shift from Th2 to Th1 chemokine association. (A) Gene expression before and after therapy was studied by NanoString and genes associated with the benign T cell population were assessed by Pearson's correlations with CD7 and CD8A (CD8 ${ }^{+} T$ cells only). CD7 was strongly correlated with the number of benign T cells as measured by HTS, validating the approach (Supplemental Figure 2). Genes associated with total benign cells had only 1 gene in common and CD8-associated genes before and after therapy were completely distinct. Genes in bold font: $r>0.9, P<0.001$. (B) PUVA induces a shift from Th2to Th1-associated chemokines. The number of benign T cells, as assessed by CD7 gene expression levels (top 2 panels), correlated strongly with the expression of the Th2-recruiting chemokine CCL18 before but not after therapy and with the Th1-recruiting chemokines CXCL9, -10, and -11 after, but not before, therapy. Similar results were seen when the number of benign T cells was assessed by HTS (bottom panel). For mRNA expression and association studies, Pearson's correlations were used to identify genes associated with CD7 or CD8A gene counts (as measured by NanoString). Genes with $r>0.8$ and $P<0.01$ were considered significant. For correlations, a Pearson's correlation coefficient with a 2-tailed $P$ value is reported. 
Supplemental Figure 4). Benign T cells expressing OX40 and malignant T cells were observed to cluster together with OX40L-expressing c-Kit ${ }^{+}$dendritic cells (Figure 6, D-F, and Figure 7D). Clusters of T cells and $\mathrm{c}-\mathrm{Kit}^{+}$cells were frequent in MF but rare in healthy skin (Supplemental Figure 12).

c-Kit ${ }^{+}$dendritic cells and malignant $T$ cells express CD4OL and CD40 is coexpressed by both malignant and benign $T$ cells in MF. Our association studies also identified CD40L as a driver of skin inflammation. CD40L is a costimulatory molecule expressed by T cells activated through the TCR and by other cell types including mast cells (18). CD40 is expressed on APCs and is also upregulated on the surface of activated $\mathrm{T}$ cells, particularly on $\mathrm{CD}^{+} \mathrm{T}$ cells. CD40 and CD40L provide independent and distinct costimulatory signals to T cells $(19,20)$. We immunostained MF skin lesions for CD40 and CD40L to identify the cell types expressing these receptors. CD40L was expressed by malignant $\mathrm{T}$ cells and by c-Kit-expressing CD11 ${ }^{+}$tryptase ${ }^{-}$dendritic cells (Figure 7, A and B, and Supplemental Figure 4), and CD40 was expressed by both benign and malignant T cells (Figure 7C). CD40 was not detectably

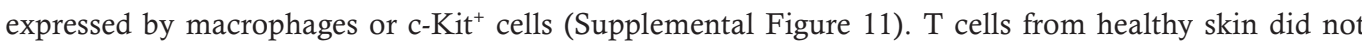
express CD40, CD40L, or OX40L (Supplemental Figure 15).

OX40/OX40L and CD40/CD40L interactions may drive inflammation via an inflammatory synapse created between $\mathrm{c}-\mathrm{Kit}^{+}$dendritic cells, malignant $\mathrm{T}$ cells, and benign $\mathrm{T}$ cells in MF. Malignant $\mathrm{T}$ cells and benign $\mathrm{T}$ cells frequently clustered around CCL18-expressing, OX40L ${ }^{+} \mathrm{CD} 40 \mathrm{~L}^{+} \mathrm{C}-\mathrm{Kit}^{+}$dendritic cells (Figure 7D and Supplemental Figure 12). We postulated that an inflammatory synapse may form between $\mathrm{c}-\mathrm{Kit}^{+}$ dendritic cells, malignant cells, and benign $\mathrm{T}$ cells and that the resulting activation of benign cells may drive visible inflammation (Figure 7E). In this model, CCL18 secreted by dendritic cells attracts Th2 benign $\mathrm{T}$ cells that are then activated by OX40/OX40L dendritic cell interactions and by CD40/ CD40L interactions provided by both dendritic and malignant $\mathrm{T}$ cells. Malignant $\mathrm{T}$ cells also receive a proinflammatory CD40/CD40L signal from dendritic cells and benign $\mathrm{T}$ cell activation may provide pro-growth signals to the tumor.

Clearance of the malignant $T$ cell clone after PUVA is associated with recruitment of new CD8 $8^{+} T$ cell clones that express markers of antigen-specific activation, are locally expanded, and may be tumor specific. PUVA eradicated malignant $\mathrm{T}$ cells from skin in $50 \%$ of the patients with less than $10 \%$ malignant $\mathrm{T}$ cells in skin before treatment, suggesting that the benign $\mathrm{T}$ cell shifts that accompany PUVA therapy may enhance tumor clearance in low-burden patients. Tumor antigens have not been identified in MF so we could not test posttreatment skin T cells for their ability to recognize the tumor. As an indirect approach, we identified $\mathrm{T}$ cell subsets that were strongly associated with markers of TCR-dependent, antigen-specific activation (CD40L, ITK, LCK, and ZAP-70) in gene expression studies. Before therapy, malignant but not benign T cells were strongly associated with markers of antigen-specific activation (Figure 8A). After therapy, benign $\mathrm{T}$ cells and $\mathrm{CD} 8^{+} \mathrm{T}$ cells in particular were strongly associated with markers of antigen reactivity. These data suggested that PUVA could bring tumor-specific T cells into the skin. If so, recruitment of $\mathrm{T}$ cells with new antigen specificities should be associated with tumor clearance. Indeed, the percentage of new $\mathrm{T}$ cell receptors recruited into the skin was strongly associated with the percentage reduction in the malignant $\mathrm{T}$ cell clone $\left(r^{2}=0.71, P<0.0001\right.$; Figure $\left.8 \mathrm{~B}\right)$. Expanded clonal populations of benign $\mathrm{T}$ cells were identified in the skin of MF patients treated with topical resiquimod and were associated with tumor clearance (15). Patients with a greater than $90 \%$ reduction in the malignant $\mathrm{T}$ cell clone had significantly more expanded benign $\mathrm{T}$ cell clones than low-responding patients (19 vs. 1.5, Figure 8C). In summary, markers of antigen-specific $\mathrm{T}$ cell activation were strongly associated with $\mathrm{T}$ cells recruited into the skin after PUVA, recruitment of T cells with new TCR specificities was strongly associated with clearance of malignant $\mathrm{T}$ cells, and expanded clonal populations of benign $\mathrm{T}$ cells were present in patients that greatly reduced or eradicated the clone. Our cohort included one exceptional high-burden patient who experienced $95 \%$ reduction in the malignant $\mathrm{T}$ cell clone after PUVA (Figure $8, \mathrm{D}-\mathrm{G})$. This patient experienced an $85 \%$ improvement in both CAILS and mSWAT clinical scores and had a marked reduction in the number of both benign and malignant $\mathrm{T}$ cells in skin after PUVA (Figure $8, \mathrm{D}$ and $\mathrm{E})$. Although the number of benign infiltrating $\mathrm{T}$ cells was markedly reduced, gene expression studies showed that markers associated with $\mathrm{CD}^{+} \mathrm{T}$ cells (CD8A) and in particular genes associated with the activated, posttreatment $\mathrm{CD}^{+}$population (GZMK, KLRK1, and CD6), were increased (Figure $8, \mathrm{~F}$ and $\mathrm{G})$. These results illustrate how recruitment of a distinct population of $\mathrm{CD}^{+} \mathrm{T}$ cells can be associated with malignant $\mathrm{T}$ cell clearance. 


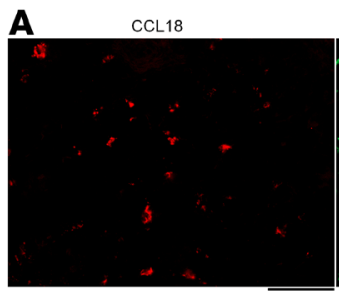

B

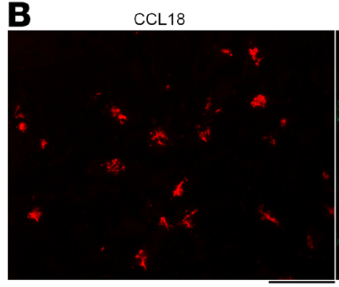

D

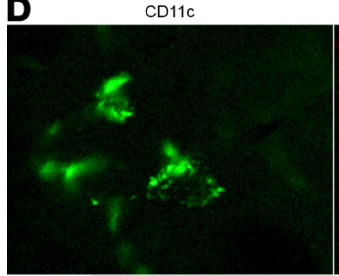

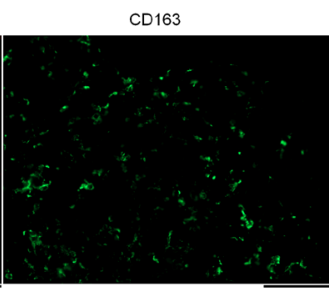

c-Kit

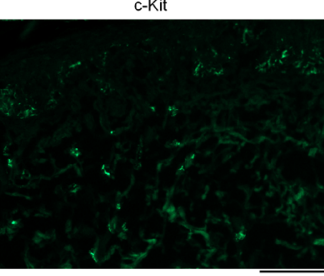

CD11c (green)/c-Kit (red)

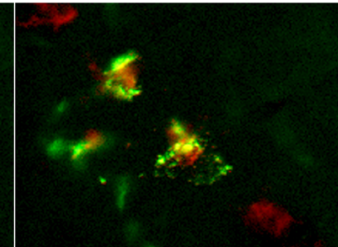

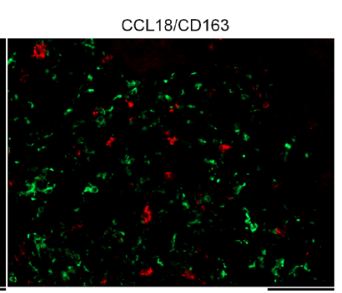

CCL18/c-Kit

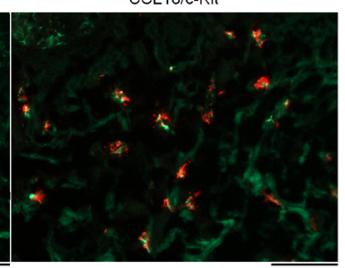

CD11C (green)/CCL18(red)

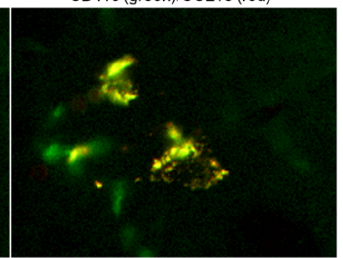

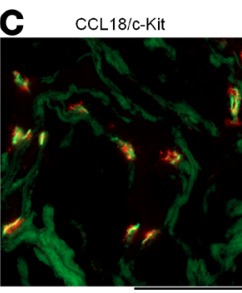

CCL18/C-Kit

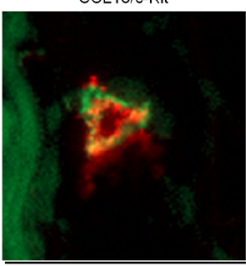

D11c (green)/c-Kit (red)/CCL18 (blue)

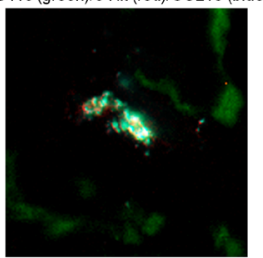

Figure 4. CCL18 is produced by c-Kit dendritic cells in mycosis fungoides (MF). (A) CD163-expressing M2-like macrophages do not produce CCL18 in MF. Coimmunostaining of CCL18 (red) and CD163 (green) demonstrated independent staining. A patient with low-burden stage IA CTCL is shown; a total of 3 stage IA-IB patients showed similar results. (B and C) CCL18 immunostaining colocalized with c-Kit-expressing dermal cells. A stage IB high-burden MF patient is shown; a total of 4 patients showed similar results. (C) Higher-power images of immunostaining of the same stage IA patient studied in $\mathbf{A}$ are shown. (D) CCL18-producing c-Kit ${ }^{+}$cells are CD11c $^{+}$dendritic cells. A stage IA high-burden patient is shown (first 3 panels) and costaining for all markers in another stage IB high-burden patient is shown (fourth panel). Triple staining appears white. Staining demonstrating that CCL18 ${ }^{+}$cells lacked expression of tryptase are included in Supplemental Figure 4. Similar results were observed in high- and low-burden patients. All lesions are untreated. Results from additional patients are shown in Supplemental Figure 5. A color blind-accessible version of this image is provided in Supplemental Figure 6. Scale bars: $100 \mu \mathrm{m}$.

\section{Discussion}

PUVA is a therapy for inflammatory skin diseases that works well in both inflammatory disorders and MF, a cancer of skin-resident $\mathrm{T}$ cells. Our studies suggest that PUVA targets the benign $\mathrm{T}$ cell population in skin. Fourteen out of 15 patients clinically improved after PUVA, in agreement with prior studies. In patients with lower than 10\% malignant $\mathrm{T}$ cell frequency, PUVA induced benign $\mathrm{T}$ cell shifts, malignant $\mathrm{T}$ cell reductions in all patients, and malignant $\mathrm{T}$ cell eradication in $50 \%$ of treated patients. $\mathrm{CD} 8^{+} \mathrm{T}$ cells bearing new antigen receptors were recruited into skin, clonally expanded in treated lesions, and were strongly associated with markers of antigen-driven activation and malignant T cell clearance. High-burden patients $(>20 \%)$ appeared to improve clinically but the frequency of malignant $\mathrm{T}$ cells increased on average after PUVA. The clinical take-home message is that PUVA is effective and potentially curative in patients with a low malignant $\mathrm{T}$ cell burden but suppresses visible inflammation without depleting malignant $\mathrm{T}$ cells in high-burden patients.

High-burden patients appeared to improve but silently harbored large numbers of malignant $\mathrm{T}$ cells in skin, suggesting that malignant $\mathrm{T}$ cells do not directly cause skin inflammation. Improved skin disease was instead linked to benign $\mathrm{T}$ cell turnover - i.e., to what percentages of $\mathrm{T}$ cell clones present before therapy were eliminated from skin. By contrast, elimination of malignant $\mathrm{T}$ cells was tightly linked to recruitment of benign $\mathrm{T}$ cells bearing new TCRs into the skin. Thus, reduced inflammation reflected the migration of old benign $\mathrm{T}$ cells out of skin and malignant $\mathrm{T}$ cell depletion depended on recruitment of new $\mathrm{T}$ cells into it.

HTS data showed an almost complete turnover of benign T cells in skin before and after PUVA therapy and expression profiling showed that benign $\mathrm{T}$ cells before and after therapy had markedly different gene associations. The number of benign $\mathrm{T}$ cells was tightly linked to levels of the Th2-recruiting chemokine CCL18 before therapy and to the Th1-recruiting chemokines CXCL9, CXCL10, and CXCL11 after therapy, suggesting a shift from Th2 to Th1 T cells after PUVA therapy. CCL18 was expressed at levels 115-fold 
A

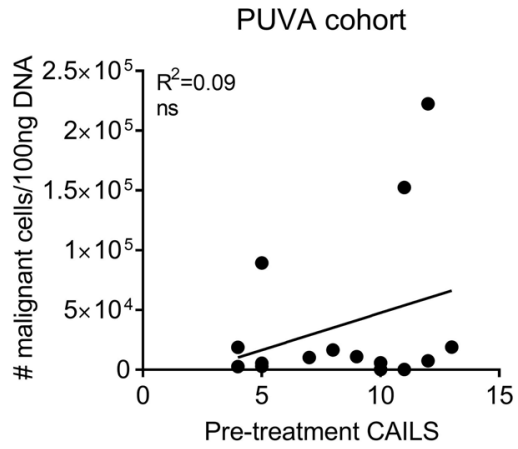

B

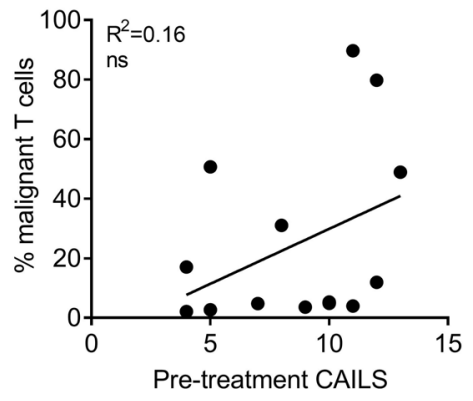

C

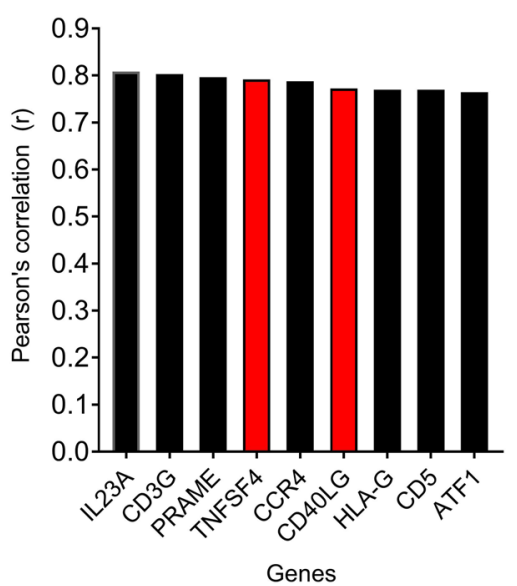

Irradiation and topical steriods cohort
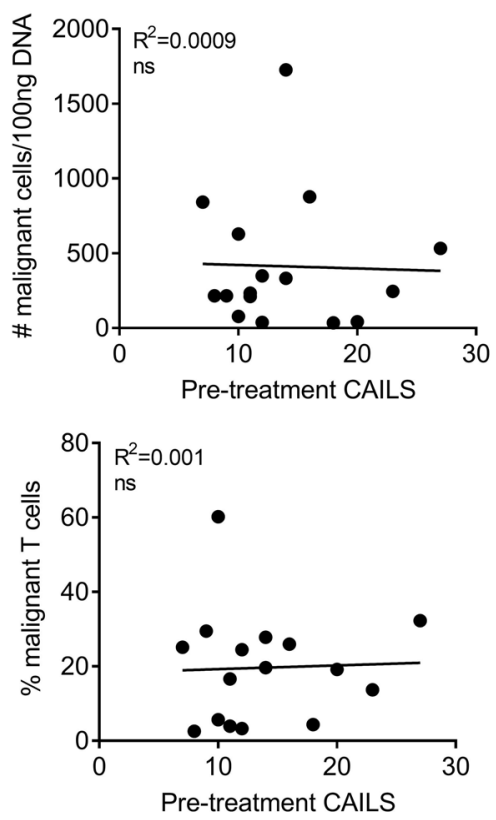

D

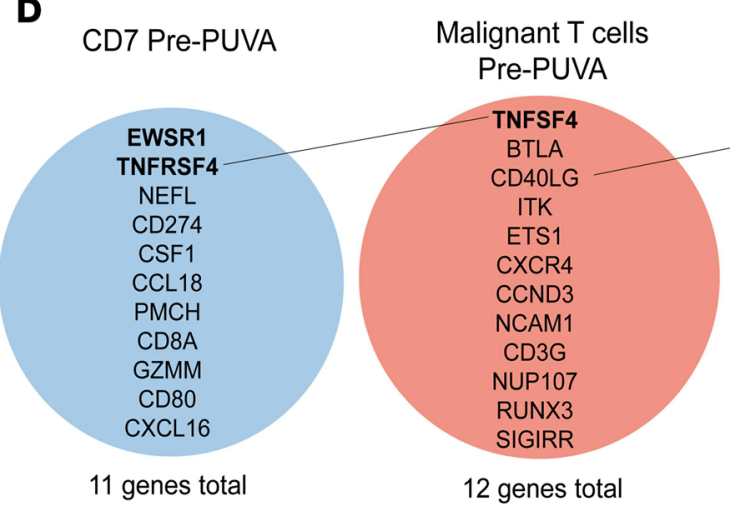

Figure 5. Skin inflammation is not linked to malignant T cell number or frequency but is related to the expression of $\mathbf{2}$ malignant T cell-associated genes. (A and B) Skin inflammation (CAILS) was not correlated with the absolute number (A) or relative percentage (B) of malignant T cells in skin in pretreatment samples from 3 clinical cohorts from 3 different institutions. (C) Genes associated with visible inflammation were identified by correlating pretreatment CAILS with genes across the PUVA treatment data set. Nine genes were identified, 3 of which (CD3G, CCR4, and CD5) are widely expressed by skin T cells and 2 of which (OX4OL and CD4OLG) were strongly associated with the number of malignant T cells in skin as measured by HTS (D).

TNFSF4 (OX40L) was strongly associated with malignant T cell numbers $(r=0.959, P=0.0006)$ and its receptor TNFRSF4 (OX40) was strongly associated with the number of benign T cells (CD7 gene expression, $r=0.971, P=0.0003$ ). Genes in bold font: $r>0.9, P<0.001$. CD40 ligand was strongly associated with malignant $\mathrm{T}$ cell number $(r=0.932, P=0.0022)$ and its ligand CD40 was associated with CD4 ${ }^{+} \mathrm{T}$ cell and macrophage (CD68, CD163) but not $\mathrm{DC}$ markers (CD11C is shown; CCL13, CCL17, CCL22, and HSD11B1 are included in Supplemental Figure 7). For correlations, a Pearson's correlation coefficient with a 2-tailed $P$ value is reported. Pearson's correlations were used to identify genes associated with pretreatment CAILS, CD7, CD40 (as measured by NanoString), or malignant T cell counts (as measured by HTS). Genes with $r>0.8$ and $P<0.01$ were considered significant.

higher in untreated MF as compared with healthy skin and was highest in low-burden patients in whom benign $\mathrm{T}$ cells outnumbered malignant $\mathrm{T}$ cells (Supplemental Figure 3). CCL18 is known to be elevated in MF and levels of CCL18 correlate with disease progression $(12,13)$.

Malignant $\mathrm{T}$ cells must ultimately drive skin inflammation; therapies that deplete malignant $\mathrm{T}$ cells lead to complete clearance of clinical inflammation and a return to normal-appearing skin (15). We identified a set of genes that were associated with the CAILS score, a measure of visible skin inflammation in the index MF lesion. Of these, OX40L and CD40L were highly correlated with the number of malignant $\mathrm{T}$ cells, suggesting they may link malignant $\mathrm{T}$ cells to inflammation. 

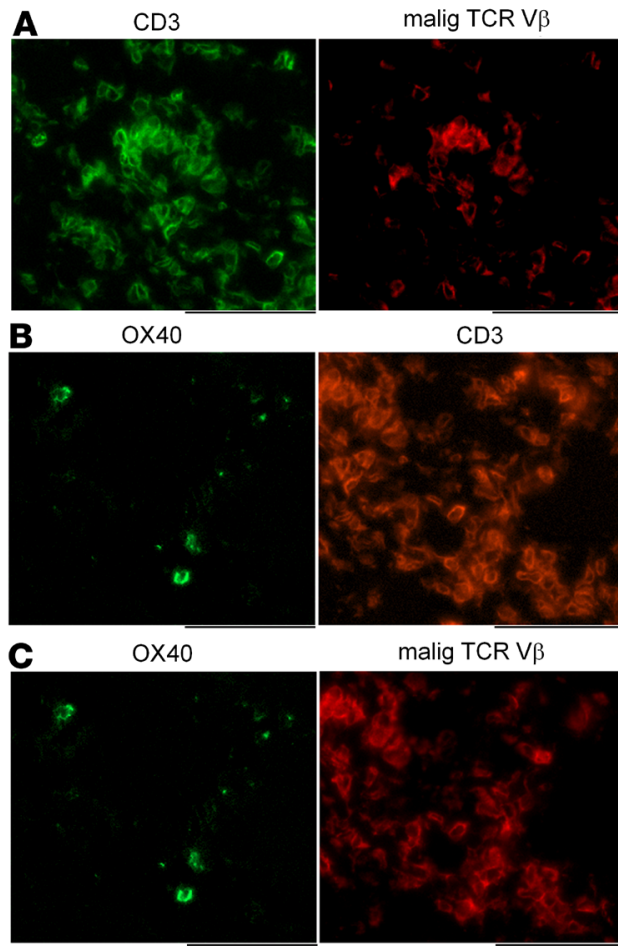

D

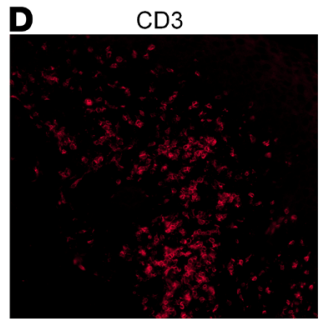

E

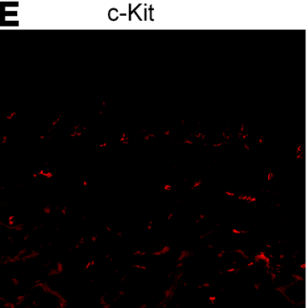

F

CD11c

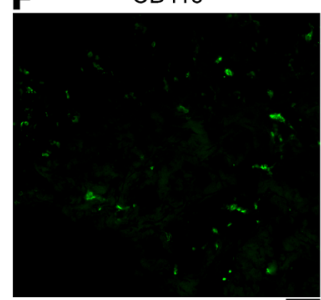

CD3

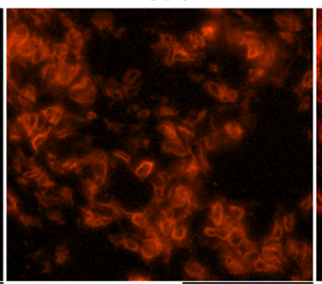

malig TCR V $\beta$

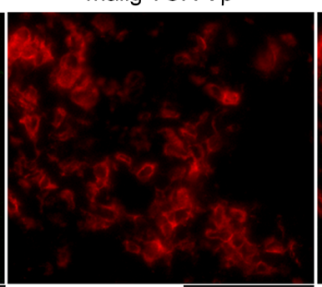

OX40L

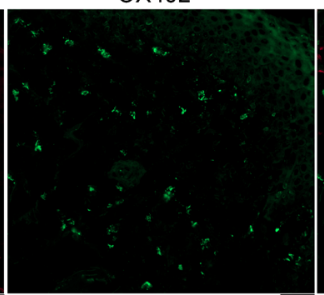

OX40L

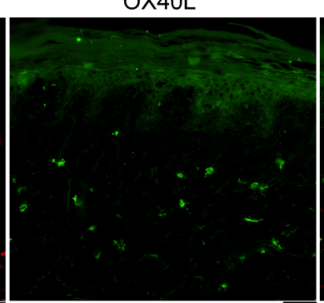

OX4OL

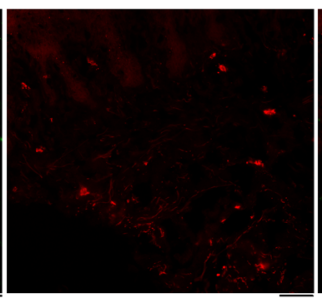

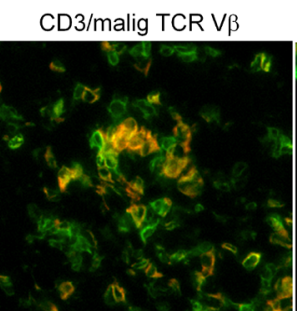

OX40/CD3

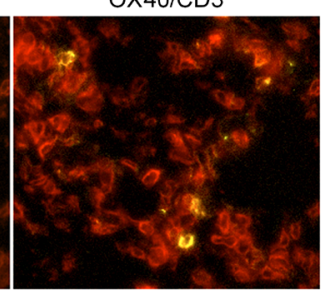

OX40/malig TCR V $\beta$

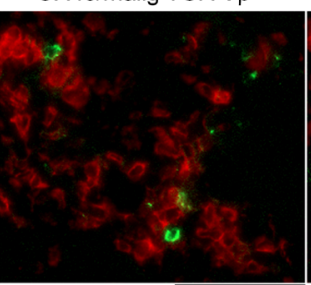

CD3/OX40L

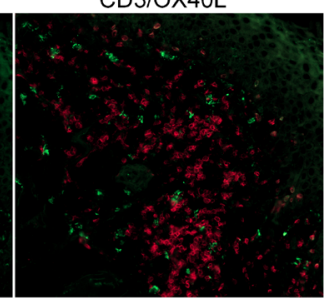

c-Kit/OX40L

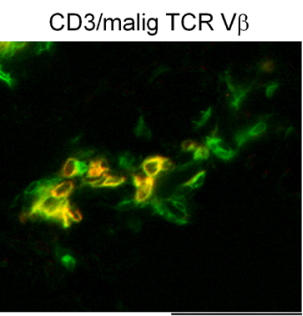

OX40/CD3

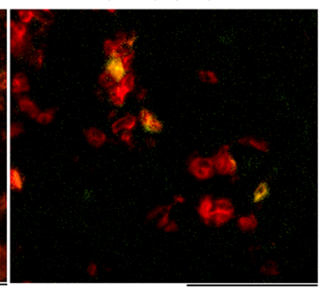

OX40/malig TCR V $\beta$

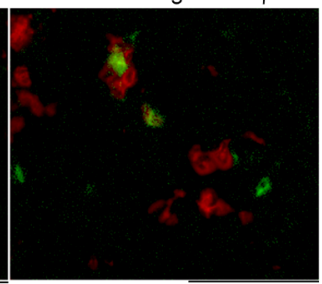

CD3/OX40L

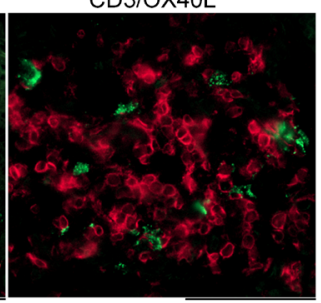

c-Kit/OX4OL

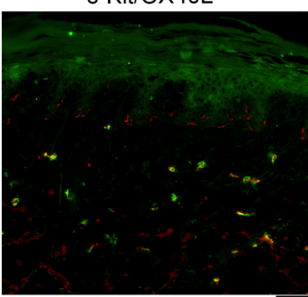

CD11c/OX40L

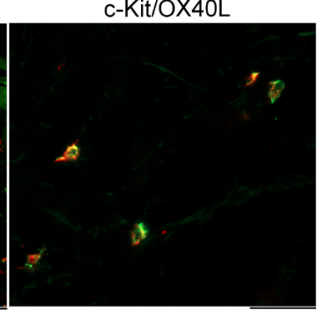

CD11c/OX40L
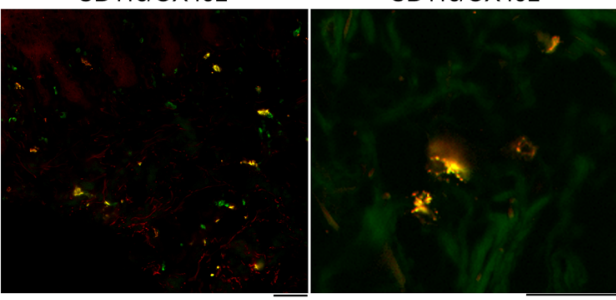

Figure 6. $0 X 40$ is expressed by benign T cells and OX40L is expressed by c-Kit ${ }^{+}$dendritic cells in mycosis fungoides (MF). (A) Malignant and benign $T$ cells can be discriminated by costaining for CD3 and the TCR V $\beta$ expressed by the malignant T cell clone, as identified by HTS. Malignant T cells costain for CD3 and the malignant T cell TCR V $\beta$ (yellow) and benign T cells stain only for CD3 (green). Stained cells from a patient with high-burden stage IIA are shown; a second field from the same donor is shown in the last panel. (B) OX40 is expressed by T cells in MF. A patient with high-burden stage IIA CTCL is shown. A second field from the same donor is shown in the last panel. Similar results were observed in a total of 3 donors. (C) OX40 is expressed by benign but not malignant T cells. OX40 and malignant T cell TCR V $\beta$ antibodies stained distinct populations of T cells. A patient with high-burden stage IIA MF is shown. A second field from the same donor is shown in the last panel. Similar results were observed in a total of 3 donors. (D) OX $40 \mathrm{~L}$ is not expressed by T cells in MF. Antibodies specific for CD3 and OX4OL stained distinct cell populations. A patient with low-burden stage IA MF is shown. The last panel is a higher magnification view of the same staining; similar results were observed in a total of 5 donors. (E) OX4OL is expressed by c-Kit ${ }^{+}$cells in MF. OX4OL and c-Kit were colocalized. Patients with high-burden stage IB (first 3 panels) and high-burden stage IA (fourth panel) MF are shown. Similar results were 
observed in a total of 3 donors. (F) OX40L-expressing cells are CD11C ${ }^{+}$dendritic cells. A patient with high-burden stage IA (first 3 panels) and a second patient with high-burden stage IB (fourth panel) MF are shown. Staining demonstrating that $\mathrm{OX} 40 \mathrm{~L}^{+}$cells lacked expression of tryptase is included in Supplemental Figure 4. All staining was performed on pretreatment skin biopsies. Results from additional patients are shown in Supplemental Figure 8. A color blind-accessible version of this image is provided in Supplemental Figure 9. Scale bars: $100 \mu \mathrm{m}$.

OX40L is a member of the tumor necrosis factor superfamily that is expressed by activated APCs and lymphocytes. OX40L binds to OX40 on T cells and transmits a costimulatory signal that promotes sustained $\mathrm{T}$ cell activation, cytokine production, division, differentiation, and survival (16). OX40 stimulation also suppresses the function and differentiation of regulatory $\mathrm{T}$ cells (21). OX40L/OX40 interactions prolong both antigen-specific and nonspecific activation of $\mathrm{T}$ cells and its net effect is to sustain chronic inflammation (16). Immunostaining demonstrated that $\mathrm{c}-\mathrm{Kit}^{+}$dendritic cells in MF expressed high levels of OX40L and that $\mathrm{T}$ cells formed clusters around $\mathrm{OX} 40 \mathrm{~L}^{+}$cells. T cells and macrophages did not express OX40L. OX40 was specifically expressed by benign but not malignant $\mathrm{T}$ cells in MF, suggesting that c-Kit ${ }^{+}$ dendritic cells provide OX40L stimulation that may drive chronic activation of benign T cells. OX40 could also recruit or retain benign $\mathrm{T}$ cells near $\mathrm{c}-\mathrm{Kit}^{+}$dendritic cells, given the finding that $\mathrm{OX} 40$ signaling drives the accumulation and retention of activated $\mathrm{CD} 4^{+} \mathrm{T}$ cells in B cell follicles (22).

$\mathrm{T}$ cells upregulate CD40L expression after encounter with a specific antigen and its binding partner CD40 is expressed by APCs, B cells, and activated CD4 ${ }^{+} \mathrm{T}$ cells (18). CD40L on T cells can drive APC maturation and $\mathrm{B}$ cell class switching, and provides a costimulatory signal to other CD40-expressing $\mathrm{T}$ cells that is as potent as CD28 signaling (19). CD40L-expressing T cells also receive a costimulatory signal from CD40L/CD40 ligation that is important for sustaining T cell responses (20). In MF, CD40L was expressed by both $\mathrm{c}-\mathrm{Kit}^{+}$dendritic cells and malignant $\mathrm{T}$ cells and CD40 was expressed by both benign and malignant $\mathrm{T}$ cells. One prior study reported CD40/CD40L coexpression by malignant T cells in MF (23). However, expression of CD40L by c-Kit ${ }^{+}$dendritic cells and CD40 by benign T cells has not been reported in MF. Our results suggest that $\mathrm{c}-\mathrm{Kit}^{+}$dendritic cells can provide costimulatory signals to both malignant and benign cells via CD40L and that $\mathrm{CD} 40 \mathrm{~L}^{+}$malignant $\mathrm{T}$ cells themselves may receive a costimulatory signal while stimulating both benign and malignant CD40-expressing T cells.

Our results show that $\mathrm{c}-\mathrm{Kit}^{+}$dendritic cells produce CCL18 that likely recruits benign Th2 T cells, creating an inflammatory synapse between dendritic cells, benign $\mathrm{T}$ cells, and malignant $\mathrm{T}$ cells. OX40/OX40L and CD40/CD40L may drive the antigen-independent activation of benign $\mathrm{T}$ cells, creating visible skin inflammation and likely providing growth factors to the tumor. $\mathrm{c}-\mathrm{Kit}^{+}$dendritic cells and benign $\mathrm{T}$ cells may also activate malignant $\mathrm{T}$ cells via CD40/CD40L interactions. MF skin lesions have been reported to contain increased numbers of predominantly immature dendritic cells (24-26), but to our knowledge, c-Kit ${ }^{+}$dendritic cells have not been reported. c-Kit ${ }^{+}$dendritic cells are induced by Th2/Th17-polarizing stimuli and act to enhance Th2/Th17 responses and to impair Th1 responses (14). In patients with gastrointestinal stromal tumors lacking c-Kit mutations, Gleevec acted on dendritic cells to block c-Kit signaling, leading to enhanced Th1 cytokine production, activation of NK cells, and enhanced antitumor immunity (27). Our studies suggest that Gleevec and inhibitors of OX40L and CD40L, in development for the treatment of autoimmunity, may have a role in the treatment of CTCL (28).

Our observation that PUVA targets benign T cells clarifies how it can treat both cutaneous lymphoma and inflammatory disorders such as psoriasis. Other antiinflammatory therapies used in CTCL also inhibit benign T cell activation. Patients with leukemic CTCL are often colonized with Staphylococcus aureus strains producing superantigens that drive the activation and proliferation of benign $\mathrm{T}$ cells; treatment of patients with antibiotics reduces cancer progression and improves survival (29, 30). An in vitro study confirmed that bacterial toxins drove the activation and cytokine production of benign $\mathrm{T}$ cells that in turn stimulated malignant $\mathrm{T}$ cell growth $(31,32)$. CTCL is the only cancer treated with extracorporeal photopheresis (ECP), an immunosuppressive therapy used to treat inflammatory disorders including allograft rejection and graft-versus-host disease (33). ECP can induce dendritic cell maturation and formation of tolerogenic dendritic cells that enhance regulatory $\mathrm{T}$ cell numbers and function $(34,35)$, presumably suppressing $\mathrm{T}$ cell inflammation in skin; it is not clear if malignant or benign $\mathrm{T}$ cells are targeted. To our knowledge, CTCL is the only cancer in which the presence of tumor-infiltrating $\mathrm{FOXP}^{+}$regulatory $\mathrm{T}$ cells is protective and associated with better outcomes (3640). Increased levels of CCL18, which we believe is responsible for recruiting protumorigenic benign 


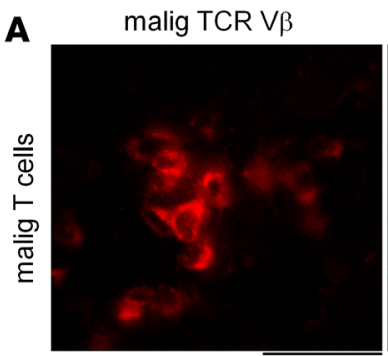

B

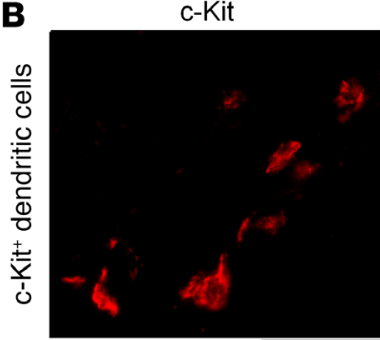

C

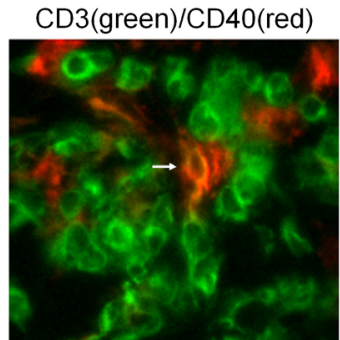

D

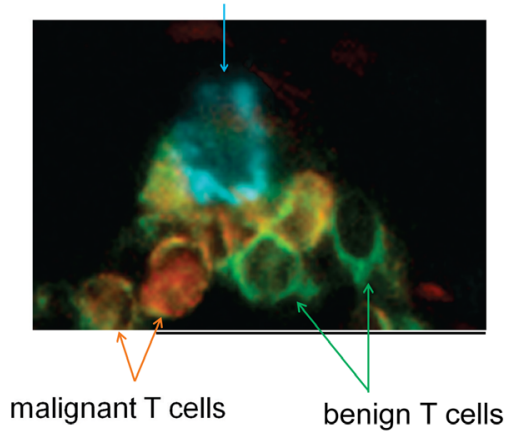

OX40L (cyan)/CD3 (green)/malig TCRV $\beta$ (red) malig TCR V $\beta / C D 40 L$

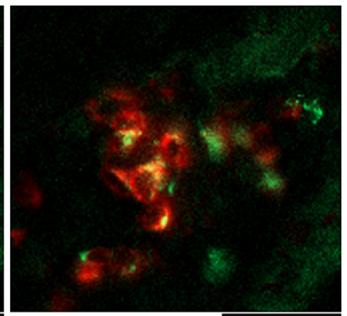

c-Kit/CD40L
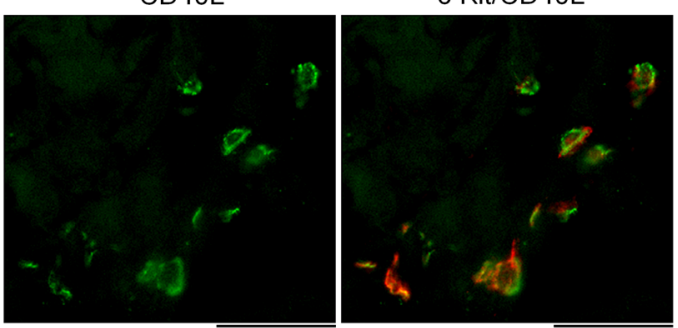

CD11c (green)/CD40L (red)
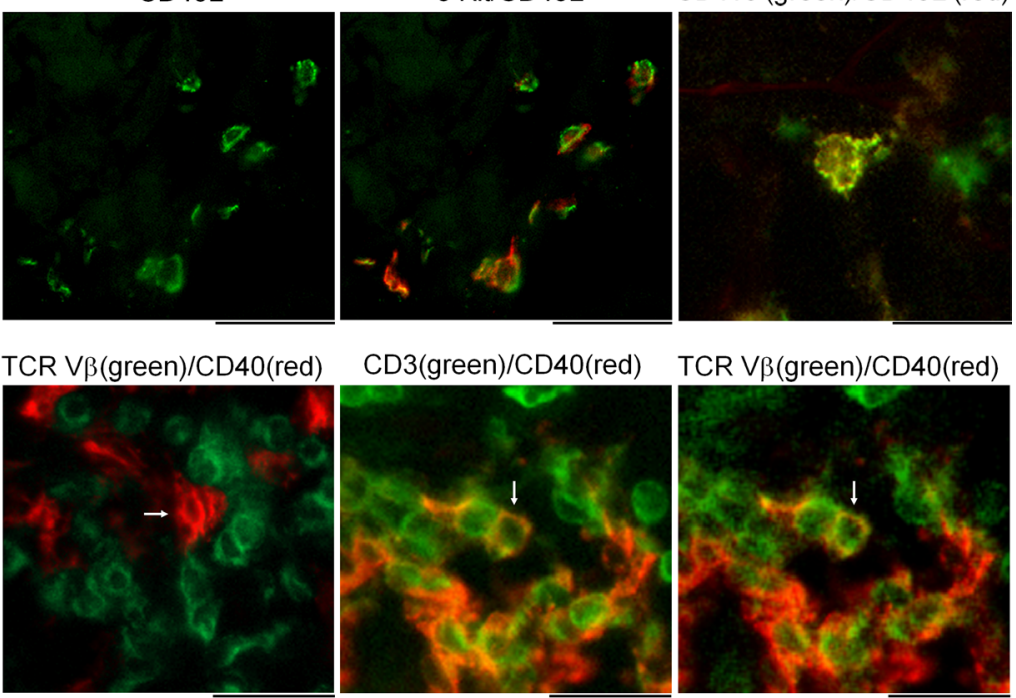

malig TCR V $\beta / C D 40 L$

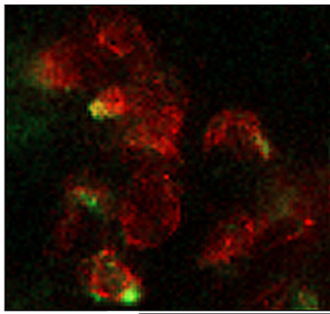

TCR V $\beta$ (green)/CD40(red)

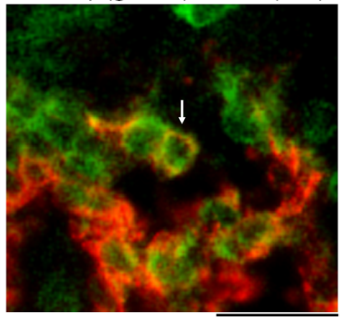

E $\mathrm{c}-\mathrm{Kit}^{+} \mathrm{OX} 40 \mathrm{~L}^{+} \mathrm{CD} 40 \mathrm{~L}^{+}$dendritic cell

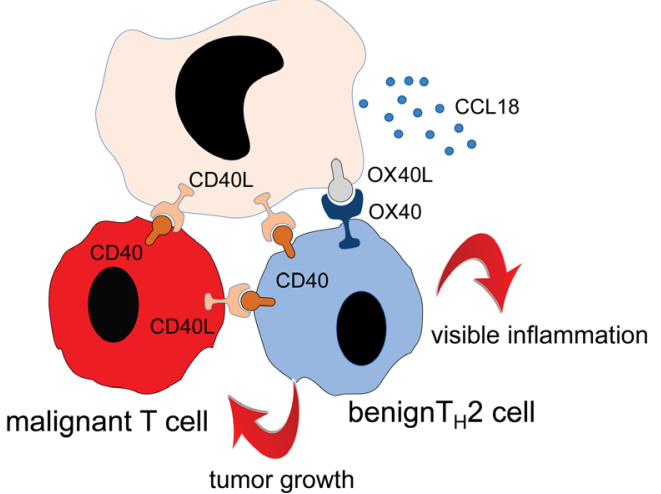

Figure 7. CD40/CD40L interactions may participate in an inflammatory synapse created between c-Kit ${ }^{+}$dendritic cells, malignant T cells, and benign T cells in mycosis fungoides. (A) CD40L is expressed by malignant T cells. A patient with high-burden stage IIA (left 3 panels) and high-burden stage IB (right panel) are shown. (B) CD4OL is also expressed by c-Kit ${ }^{+}$dendritic cells. A patient with low-burden stage IA (left 3 panels) CTCL is shown; similar results were observed in a total of 3 donors. Costaining of CD4OL ${ }^{+}$cells with the dendritic cell marker CD11c is shown in the right panel. (C) Both benign and malignant T cells express CD40. The left 2 panels show benign T cells (CD3+V $\beta^{-}$) expressing CD40. The right 2 panels show malignant T cells $\left(C D 3^{+} V \beta^{+}\right)$expressing CD40. Both fields are from the same high-burden stage IIA donor; similar results were observed in a total of 3 donors. (D) An inflammatory synapse is created between dendritic cells, malignant T cells, and benign T cells. Clusters of dendritic cells, benign T cells, and malignant T cells were frequently observed in MF, as illustrated by this costain for OX40L dendritic cells, CD3, and the malignant TCR V $\beta$. A patient with high-burden stage IIB is shown; clusters of these cell types were observed in 5 donors. (E) A proposed model for c-Kit ${ }^{+}$dendritic cell, benign T cell, and malignant T cell interactions in MF. Benign T cells are recruited by c-Kit ${ }^{+}$dendritic cell-produced CCL18 and activated by OX40/OX40L and CD40/CD40L interactions leading to visible skin inflammation and protumorogenic signals. Dendritic cells may also directly stimulate malignant T cells via CD40/ CD40L interactions. All staining was performed on pretreatment skin biopsies. Results from additional patients are shown in Supplemental Figure 13. A color blind-accessible version of this image is provided in Supplemental Figure 14. Scale bars: $50 \mu \mathrm{m}$. 


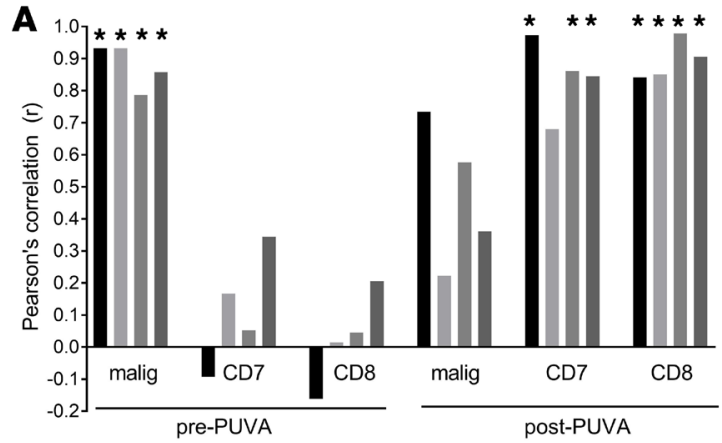

D

Pt 5: 85\% improved CAILS, $85 \%$ improved mSWAT

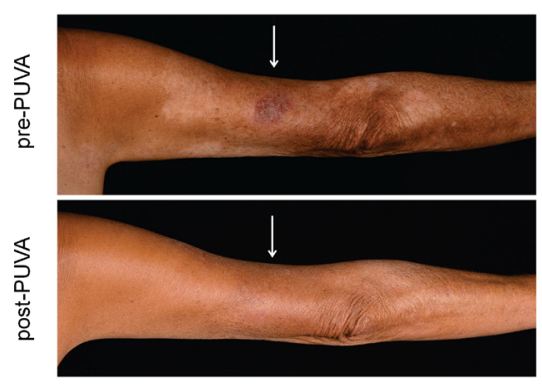

B

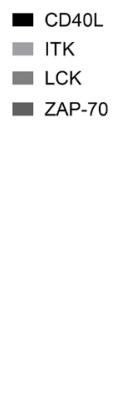

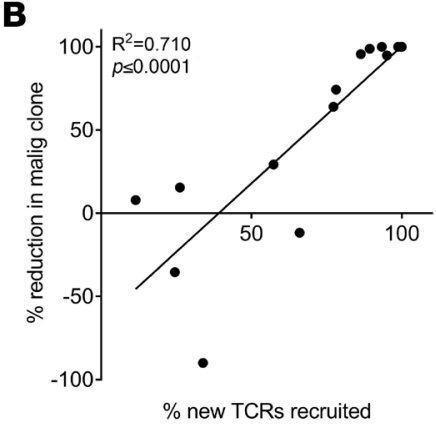

$\%$ new TCRs recruited

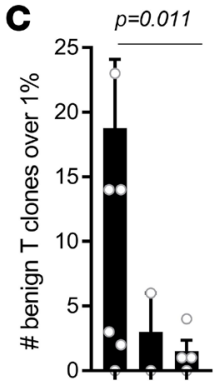

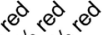

F

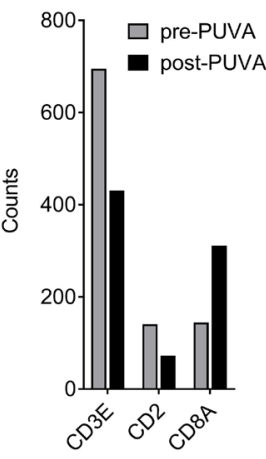

G

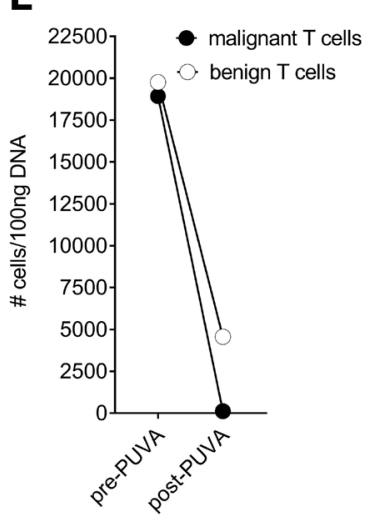

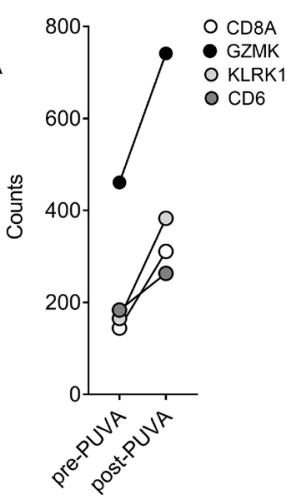

Figure 8. Clearance of the malignant T cell clone after PUVA is associated with recruitment of new CD8+ $T$ cell clones that express markers of antigenspecific activation, are locally expanded, and may be tumor specific. (A) Markers of TCR-dependent, antigen-specific activation (CD4OL, ITK, LCK, and ZAP-70) are strongly associated with malignant T cells before but not after therapy and are strongly associated with benign and CD8 ${ }^{+} \mathrm{T}$ cells after, but not before, therapy. Expression of these genes was correlated with malignant T cell number (assessed by HTS), benign T cells (CD7 gene), and CD8 ${ }^{+} \mathrm{T}$ cells (CD8A gene) before and after therapy. ${ }^{*} P<0.05$. (B) The recruitment of new $T$ cell receptors into the tumor is strongly associated with clearance of the malignant T cell clone. The percentage of total T cell clones bearing antigen receptors not seen before therapy is shown on the $x$ axis and the percentage reduction in the malignant T cell clone is shown on the $y$ axis. A Pearson's correlation coefficient with a 2-tailed $P$ value is reported. (C) Patients who eradicated or greatly reduced malignant T cells had expanded clonal populations of benign T cells after therapy. The number of expanded benign T cell clones making up greater than $1 \%$ of total benign T cells after therapy are shown for patients who had a greater than $90 \%$ reduction (>90\% red), a $50 \%$ $90 \%$ reduction ( $50-90 \%$ red), or less than a 50\% reduction ( $<50 \%$ red) in malignant T cells after PUVA therapy. The mean and SEM are shown; a KruskalWallis 1-way analysis of variance with a Bonferroni-Dunn post hoc test for multiple means testing was used $(\alpha=0.05)$. The $P$ value is adjusted for multiple comparison testing. (D-F) An illustrative patient (Pt 5) is shown who had marked reductions in clinical inflammation (D), malignant and total benign T cell numbers in skin (E), but an increase in $\mathrm{CD}^{+} \mathrm{T}$ cell-associated genes ( $\mathbf{F}$ and $\left.\mathbf{G}\right)$. Malignant and benign T cell numbers were determined by HTS $(\mathbf{E})$ and expression counts for T cell-associated (CD3 and CD2) and CD8-associated (CD8A, GZMK, KLRK1, and CD6) genes (F and G) were measured by NanoString.

Th2 T cells, is associated with tumor progression and worse outcomes in CTCL. Taken together, these results suggest that benign $\mathrm{T}$ cells provide critical growth signals to malignant $\mathrm{T}$ cells and that depriving malignant $\mathrm{T}$ cells of these signals not only makes patients look and feel better, it can decrease disease progression and improve survival.

There are drawbacks to our experimental approach and many questions remain unanswered. Because we had access only to frozen skin specimens, we could not isolate viable immune cells from skin and study them directly. Tumor antigens have not been identified in MF so we were compelled to rely on indirect measures of antigen specificity and were not able to demonstrate conclusively that responding $\mathrm{CD} 8^{+} \mathrm{T}$ cells in low-burden patients after PUVA were tumor specific. We have not identified how malignant $\mathrm{T}$ cells recruit and activate $\mathrm{c}-\mathrm{Kit}^{+}$dendritic cells and little is known about the effect of PUVA on dendritic cells in MF. The protumorigenic signals provided by benign $\mathrm{T}$ cell activation have not been identified. Lastly, malignant $\mathrm{T}$ cells before treatment are strongly associated with markers of $\mathrm{T}$ cell-specific activation. This suggests that malignant $\mathrm{T}$ cells are either driven by antigen exposure or the TCR signaling pathway is uncoupled from the TCR, as has been demonstrated in leukemic CTCL (41). These unanswered questions are the subject of ongoing studies in our laboratory. 
In summary, our studies demonstrate that visible skin inflammation is driven by benign, not malignant, $\mathrm{T}$ cells in MF. PUVA induces shifts in the benign $\mathrm{T}$ cell population that both reduce visible inflammation and recruit new benign $\mathrm{T}$ cells capable of eradicating malignant $\mathrm{T}$ cells in low- but not high-burden patients. Our work provides evidence for an inflammatory synapse between malignant and benign $\mathrm{T}$ cells and $\mathrm{c}-\mathrm{Kit}^{+}$dendritic cells. Targeting $\mathrm{c}-\mathrm{Kit}^{+}$dendritic cell and benign $\mathrm{T}$ cell recruitment and activation may be novel therapeutic avenues for the treatment of MF.

\section{Methods}

Study design and skin samples. This is an experimental laboratory study performed on human tissue samples. Patients who were studied met the World Health Organization-European Organization for Research and Treatment of Cancer (WHO-EORTC) criteria for MF (1). Biopsy specimens from 15 stage IA-IIA MF patients before and after 12 to 15 weeks of PUVA therapy were obtained from patients treated at the Medical University of Graz. Patients received $10 \mathrm{mg} / 20 \mathrm{~kg}$ 8-methoxypsoralen 1 hour prior to twice weekly UVA exposure. UVA was administered below $50 \%$ of the minimal phototoxic dose at start of treatment. UVA dose was increased weekly by $0 \%-30 \%$, depending on the absence or presence of erythema after preceding treatments. Evaluation of efficacy was based on improvement from baseline of the CAILS score, which evaluates changes from baseline of the treated target lesions for size, erythema, scaling, and lesion elevation and on change in baseline of the mSWAT score, which evaluates the entire skin surface for percentage involvement with a weighted score based on whether a lesion is a patch, plaque, or tumor. Lesional skin biopsies from 10 patients with stage IA-IIA MF before treatment with topical resiquimod were obtained from the University of Pennsylvania (15). Lesional skin biopsies from 16 patients with stage IA-IIA MF were obtained prior to therapy with low-dose radiation or topical steroids from patients seen at the Dana-Farber/Brigham and Women's Cancer Center Cutaneous Lymphoma Program. Skin from healthy individuals was obtained from healthy patients undergoing cosmetic surgery procedures. Immunostaining and gene expression studies were performed using in vitro assays without blinding or randomization. Study components were not predefined.

DNA isolation and HTS. DNA was isolated from formalin-fixed, paraffin-embedded or frozen, OCT-embedded skin samples and studied by ImmunoSEQ (Adaptive Biotechnologies) using 100-400 ng of DNA template as previously described (17). The percentage of $\mathrm{T}$ cells consisting of the malignant clone was determined by dividing the abundance of the malignant clone (number of reads) by the total number of $\mathrm{T}$ cell reads. The number of malignant $\mathrm{T}$ cells per sample was obtained by multiplying the malignant clone frequency and the total number of productive templates. To normalize the samples based on total input DNA, we divided the number of T cells/100 ng input DNA.

$R N A$ isolation and quantification. RNA was isolated from healthy skin and CTCL skin samples using the RNeasy FFPE or RNeasy Mini Kit (Qiagen), respectively, as per the manufacturer's instructions. The expression of 770 inflammation-related genes was measured using the NanoString Human PanCancer Immune Profiling Panel. Normalization and analysis of NanoString data were carried out using nSolver software. Normalization factors were calculated based on 40 reference genes.

Cryosection immunostaining. Five-micrometer cryosections were cut from OCT-embedded skin samples. Slides were fixed in acetone, rehydrated in 1× TBS/saponin (Boston BioProducts), blocked with $0.017 \mu \mathrm{g} /$ $\mu 1$ human IgG, and then stained with relevant antibodies in $1 \times$ TBS/saponin for 30 minutes, with 10-minute wash steps between antibodies as necessary. Slides were washed after antibody treatment and mounted in Vectashield Hard Set Mounting Medium with DAPI (Vector Labs). Tissues were imaged immediately after mounting on a Mantra Quantitative Pathology Workstation using Mantra Snap 1.0 imaging software and analyzed with inForm image analysis software (PerkinElmer). Antibodies and the concentrations used are listed in Supplemental Table 4.

Statistical analyses. Primary methods of data analysis included descriptive statistics (means, medians, and standard deviations). Differences between 2 sample groups were detected using the 1-tailed Wilcoxon-Mann-Whitney test $(\alpha=0.05)$. For comparisons of multiple groups, a Kruskal-Wallis 1 -way analysis of variance with a Bonferroni-Dunn post hoc test for multiple means testing was used $(\alpha=0.05)$. All reported $P$ values are adjusted for multiple comparison testing. For mRNA expression and association studies, Pearson's correlations were used to identify genes associated with malignant T cells (number of cells/100 ng DNA by HTS), or with CD7 or CD8A gene counts (as measured by NanoString). Only genes with $r>0.8$ and $P<0.01$ were considered significant. 
Study approval. All studies were performed in accordance with the Declaration of Helsinki. Written consent was obtained from all patients before study entry and sample collection. All tissues were collected with previous approval from the relevant review boards: Medical University of Graz Ethical Committee, University of Pennsylvania's IRB, and the Dana-Farber Cancer Institute's IRB. Translational studies were approved by the IRB of the Partners Human Research Committee. Written informed consent was provided for clinical images appearing in the manuscript.

\section{Author contributions}

RAC and PW designed the experiments. RAC supervised experiments, analyzed data, carried out statistical analyses, drafted figures, and wrote and revised the manuscript. PVG, JTO, CHY, JDC, EWS, JET, AMV, AG, ZY, and ELL carried out experiments, analyzed data, and carried out statistical analyses. JET also edited the manuscript. TSW provided expertise on gene expression analyses. JIN assisted with references. PW, JTO, and AHR designed the clinical studies, oversaw the clinical treatment of patients, and provided skin specimens. PW designed and carried out the clinical trial of PUVA in CTCL patients on which these translational studies are based.

\section{Acknowledgments}

The authors would like to thank the patients who made this work possible, for entrusting us with their clinical care, and for donating skin samples. Andrea Troxel (New York University Langone Medical Center) provided statistical advice. Thomas Cochran of the Boston Center for Plastic Surgery and Bohdan Pomahac, Simon Talbot, and Elof Eriksson of Brigham and Women's Hospital generously provided healthy adult human skin samples. Regina Fink-Puches, Alexandra Gruber-Wackernagel, Angelika Hofer, and Franz Legat of Medical University of Graz were responsible for clinical care of patients treated with PUVA. This work was supported by NIH/National Institute of Arthritis and Musculoskeletal and Skin Diseases (NIAMS) grants R01 AR063962 (to RAC) and R01 AR074797 (to RAC and AHR); NIH/National Cancer Institute (NCI) R01 CA203721 (to RAC); NIH T32 AR-07098-36 (supplied salary for JTO and CHY); the KL2/Catalyst Medical Research Investigator Training (CMeRIT) program (supplied salary for JTO); NIH/ NCI R01 CA122569 (to AR); a Translational Research grant from the Leukemia and Lymphoma Society (to AR); a CLARIONS grant from the Cutaneous Lymphoma Foundation (to RAC); as well as FWF Austrian Science Fund no. W1241, the Oesterreichische Nationalbank Anniversary Fund no.15463, the Austrian Society of Dermatology and Venereology (all to $\mathrm{PW}$ ); and $\mathrm{PhD}$ program Molecular Fundamentals of Inflammation (MOLIN), the Medical University of Graz, Austria (supplied salary to PVG).

Address correspondence to: Rachael A. Clark, Department of Dermatology, Brigham and Women's Hospital, Room 501A, 221 Longwood Avenue, Boston, Massachusetts 02115, USA. Phone: 617.525.8502; Email: rclark@bwh.harvard.edu. Or to: Peter Wolf, Research Unit for Photodermatology, Department of Dermatology and Venereology, Medical University of Graz, Auenbruggerplatz 8, 8036 Graz, Austria. Phone: 43.316.385.12371; Email: peter.wolf@medunigraz.at.

1. Willemze R, et al. WHO-EORTC classification for cutaneous lymphomas. Blood. 2005;105(10):3768-3785.

2. Campbell JJ, Clark RA, Watanabe R, Kupper TS. Sezary syndrome and mycosis fungoides arise from distinct T-cell subsets: a biologic rationale for their distinct clinical behaviors. Blood. 2010;116(5):767-771.

3. Clark RA, et al. Skin effector memory T cells do not recirculate and provide immune protection in alemtuzumab-treated CTCL patients. Sci Transl Med. 2012;4(117):117ra7.

4. Kim YH, Liu HL, Mraz-Gernhard S, Varghese A, Hoppe RT. Long-term outcome of 525 patients with mycosis fungoides and Sezary syndrome: clinical prognostic factors and risk for disease progression. Arch Dermatol. 2003;139(7):857-866.

5. Honig B, Morison WL, Karp D. Photochemotherapy beyond psoriasis. J Am Acad Dermatol. 1994;31(5 Pt 1):775-790.

6. Pathak MA, Fitzpatrick TB. The evolution of photochemotherapy with psoralens and UVA (PUVA): 2000 BC to 1992 AD. J Photochem Photobiol B, Biol. 1992;14(1-2):3-22.

7. Olsen EA, et al. Guidelines for phototherapy of mycosis fungoides and Sézary syndrome: A consensus statement of the United States Cutaneous Lymphoma Consortium. J Am Acad Dermatol. 2016;74(1):27-58.

8. Querfeld C, et al. Long-term follow-up of patients with early-stage cutaneous T-cell lymphoma who achieved complete remission with psoralen plus UV-A monotherapy. Arch Dermatol. 2005;141(3):305-311.

9. Weinstock MA, Gardstein B. Twenty-year trends in the reported incidence of mycosis fungoides and associated mortality. Am J Public Health. 1999;89(8):1240-1244.

10. Ormsby A, Bergfeld WF, Tubbs RR, Hsi ED. Evaluation of a new paraffin-reactive CD7 T-cell deletion marker and a polymerase chain reaction-based T-cell receptor gene rearrangement assay: implications for diagnosis of mycosis fungoides in com- 
munity clinical practice. J Am Acad Dermatol. 2001;45(3):405-413.

11. Michie SA, Abel EA, Hoppe RT, Warnke RA, Wood GS. Discordant expression of antigens between intraepidermal and intradermal T cells in mycosis fungoides. Am J Pathol. 1990;137(6):1447-1451.

12. Günther C, et al. Up-regulation of the chemokine CCL18 by macrophages is a potential immunomodulatory pathway in cutaneous T-cell lymphoma. Am J Pathol. 2011;179(3):1434-1442.

13. Miyagaki T, et al. Increased CCL18 expression in patients with cutaneous T-cell lymphoma: association with disease severity and prognosis. J Eur Acad Dermatol Venereol. 2013;27(1):e60-e67.

14. Krishnamoorthy N, et al. Activation of c-Kit in dendritic cells regulates T helper cell differentiation and allergic asthma. Nat Med. 2008;14(5):565-573.

15. Rook AH, et al. Topical resiquimod can induce disease regression and enhance T-cell effector functions in cutaneous T-cell lymphoma. Blood. 2015;126(12):1452-1461.

16. Redmond WL, Ruby CE, Weinberg AD. The role of OX40-mediated co-stimulation in T-cell activation and survival. Crit Rev Immunol. 2009;29(3):187-201.

17. Kirsch IR, et al. TCR sequencing facilitates diagnosis and identifies mature T cells as the cell of origin in CTCL. Sci Transl Med. 2015;7(308):308ra158.

18. Grewal IS, Flavell RA. The role of CD40 ligand in costimulation and T-cell activation. Immunol Rev. 1996;153:85-106.

19. Munroe ME, Bishop GA. A costimulatory function for T cell CD40. J Immunol. 2007;178(2):671-682.

20. Howland KC, Ausubel LJ, London CA, Abbas AK. The roles of CD28 and CD40 ligand in T cell activation and tolerance. J Immunol. 2000;164(9):4465-4470.

21. Vu MD, et al. OX40 costimulation turns off Foxp3+ Tregs. Blood. 2007;110(7):2501-2510.

22. Brocker T, Gulbranson-Judge A, Flynn S, Riedinger M, Raykundalia C, Lane P. CD4 T cell traffic control: in vivo evidence that ligation of OX40 on CD4 T cells by OX40-ligand expressed on dendritic cells leads to the accumulation of CD4 T cells in B follicles. Eur J Immunol. 1999;29(5):1610-1616.

23. Storz M, Zepter K, Kamarashev J, Dummer R, Burg G, Häffner AC. Coexpression of CD40 and CD40 ligand in cutaneous T-cell lymphoma (mycosis fungoides). Cancer Res. 2001;61(2):452-454.

24. Lüftl M, Feng A, Licha E, Schuler G. Dendritic cells and apoptosis in mycosis fungoides. Br J Dermatol. 2002;147(6):1171-1179.

25. Schlapbach C, Ochsenbein A, Kaelin U, Hassan AS, Hunger RE, Yawalkar N. High numbers of DC-SIGN ${ }^{+}$dendritic cells in lesional skin of cutaneous T-cell lymphoma. J Am Acad Dermatol. 2010;62(6):995-1004.

26. Schwingshack1 P, Obermoser G, Nguyen VA, Fritsch P, Sepp N, Romani N. Distribution and maturation of skin dendritic cell subsets in two forms of cutaneous T-cell lymphoma: mycosis fungoides and Sézary syndrome. Acta Derm Venereol. 2012;92(3):269-275.

27. Borg C, et al. Novel mode of action of c-kit tyrosine kinase inhibitors leading to NK cell-dependent antitumor effects. $J$ Clin Invest. 2004;114(3):379-388.

28. Redmond WL, Weinberg AD. Targeting OX40 and OX40L for the treatment of autoimmunity and cancer. Crit Rev Immunol. 2007;27(5):415-436.

29. Tokura Y, et al. Cutaneous colonization with staphylococci influences the disease activity of Sézary syndrome: a potential role for bacterial superantigens. Br J Dermatol. 1995;133(1):6-12.

30. Jackow CM, Cather JC, Hearne V, Asano AT, Musser JM, Duvic M. Association of erythrodermic cutaneous T-cell lymphoma, superantigen-positive Staphylococcus aureus, and oligoclonal T-cell receptor V beta gene expansion. Blood. 1997;89(1):32-40.

31. Woetmann A, et al. Nonmalignant T cells stimulate growth of T-cell lymphoma cells in the presence of bacterial toxins. Blood 2007;109(8):3325-3332.

32. Willerslev-Olsen A, et al. Bacterial toxins fuel disease progression in cutaneous T-cell lymphoma. Toxins (Basel). 2013;5(8):1402-1421.

33. Oliven A, Shechter Y. Extracorporeal photopheresis: a review. Blood Rev. 2001;15(2):103-108.

34. Berger C, et al. Rapid generation of maturationally synchronized human dendritic cells: contribution to the clinical efficacy of extracorporeal photochemotherapy. Blood. 2010;116(23):4838-4847.

35. Lamioni A, et al. The immunological effects of extracorporeal photopheresis unraveled: induction of tolerogenic dendritic cells in vitro and regulatory T cells in vivo. Transplantation. 2005;79(7):846-850.

36. Klemke CD, et al. Paucity of FOXP3 $3^{+}$cells in skin and peripheral blood distinguishes Sézary syndrome from other cutaneous T-cell lymphomas. Leukemia. 2006;20(6):1123-1129.

37. Gjerdrum LM, et al. FOXP3 ${ }^{+}$regulatory T cells in cutaneous T-cell lymphomas: association with disease stage and survival Leukemia. 2007;21(12):2512-2518.

38. Kasprzycka M, et al. Gamma c-signaling cytokines induce a regulatory T cell phenotype in malignant CD4 ${ }^{+} \mathrm{T}$ lymphocytes. J Immunol. 2008;181(4):2506-2512.

39. Solomon GJ, Magro CM. Foxp3 expression in cutaneous T-cell lymphocytic infiltrates. J Cutan Pathol. 2008;35(11):1032-1039.

40. Fujimura T, Okuyama R, Ito Y, Aiba S. Profiles of Foxp3 ${ }^{+}$regulatory T cells in eczematous dermatitis, psoriasis vulgaris and mycosis fungoides. Br J Dermatol. 2008;158(6):1256-1263.

41. Wang L, et al. Genomic profiling of Sézary syndrome identifies alterations of key T cell signaling and differentiation genes. Nat Genet. 2015;47(12):1426-1434. 